\title{
Stimulus und Kognition. Zur Aktivierung mentaler Raumbilder*
}

\author{
Alfred Lameli/Christoph Purschke/Roland Kehrein (Marburg)
}

\begin{abstract}
Within the context of investigations of cognitive spatial models, the study analyzes the effect of varying the informational structure of linguistic survey instruments. A group of informants was asked to draw German language areas on different types of geographic maps. First, in a quantitative approach we show how the specific informational structure of each map type influences the frequency and prominence of the named language areas. The second part of the study focuses on the localization of the mentioned language areas. Here we discuss the specific effects of each type of map and the information it contains on the localization. We also discuss general characteristics of the conceptualization of linguistic space as well as differences in the perception of the prominent language areas.
\end{abstract}

\section{$1 \quad$ Einleitung}

Die Erforschung regionaler Sprachvariation ist zumeist ausgerichtet auf die formale Beschreibung und Interpretation sprachlicher Formen im Raum, definiert also eine objektgerichtete Perspektive auf das Zusammenwirken von sprachlicher Variabilität und Geographie. Nahezu sämtliche Dialektatlanten des deutschsprachigen Raumes liefern diesbezüglich ein beredtes Zeugnis. Subjektive Daten hingegen werden in der Regel nicht auf den Raum, sondern auf die individuelle Disposition von Sprechern (Einstellungen, Bewertungen, ...) bezogen, zumeist in Form von punktuellen Analysen (z. B. Siebenhaar 2000). ${ }^{1}$ In den Kognitionswissenschaften, allen voran der Psychologie, aber etwa auch im Rahmen einer kognitiv orientierten Geographie, ist dagegen spätestens seit den 1970er Jahren die Frage nach der mentalen Repräsentation räumlicher Strukturen im Zusammenhang mit kartographischen Darstellungsmitteln vielfach diskutiert worden und darf dort als intradisziplinär etablierter Schwerpunkt betrachtet werden (cf. z. B. Gould/White 1986, Kitchin 1994, Downs/Stea 1973). Eines der Standardverfahren zum Beleg mentaler Repräsentationen ist dabei die Erhebung sogenannter mental maps (auch als cognitive maps bezeichnet). ${ }^{2}$ Um solche Konzepte aufzudecken, werden Informanten gebeten, bestimmte räumliche Aspekte ihres individuellen Erfahrungsbereichs zu visualisieren.

Wesentlich durch angloamerikanischen Einfluss angestoßen wurde auch in der Variationslinguistik der vergangenen Jahre verstärkt auf die den traditionellen Zugang ergänzenden Möglichkeiten einer solchen, kognitiv motivierten Erschließung der Sprecher- und Hörerperspektive hingewiesen. Im deutschsprachigen Raum ist aktuell starkes Interesse an einer als "perceptual dialectology" (Preston 1999, Niedzielsky/Preston 2000) firmierenden Ausrichtung festzustellen (z. B. Gessinger 2000, Auer 2004, Hofer 2004, Hundt/Anders i. E.). Da hier jedoch abgesehen von einzelnen Ansätzen (Diercks 1988, Schmitt 1992) keine dezidierte

\footnotetext{
* Die Autoren danken den anonymen Gutachtern der "Linguistik online" für ihre Anmerkungen.

${ }^{1}$ Eine ausführliche Diskussion der unterschiedlichen methodischen Ansätze zur Erhebung von Einstellungs- und Bewertungsdaten gegenüber sprachlicher Variation findet sich in Purschke (i. V.).

2 Wesentlich beeinflusst wurde das Konzept der "cognitive maps" durch Tolman (1948).
} 
Forschungstradition besteht und darüber hinaus sprachliche Variation im Deutschen grundsätzlich anderen Bedingungen unterliegt als etwa in den USA, wäre eine Methodendiskussion ebenso wünschenswert wie erforderlich. Deutlich wird dies gerade auch an der Exploration von Mental Maps, die gegenwärtig einen Schwerpunkt der deutschsprachigen Forschung ausmachen. Bei genauer Betrachtung sieht man sich mit einer hohen methodischen Variation konfrontiert, die nicht zuletzt die Ausarbeitung der Erhebungsinstrumente betrifft: Weist das angloamerikanische Vorbild Prestons in einer den Informanten bereitgestellten Grundkarte lediglich die Grenzen der einzelnen Bundesstaaten auf, so verwendet z. B. Hofer (2004) eine Blankovorlage, Auer (2004) präsentiert Karten mit einer hohen Informationsdichte (Staatsgrenze, Bundesländergrenzen, Städte, Flüsse, Verkehrswege), Hundt/Anders (i. E.) verwenden eine Karte, die neben den Staatsgrenzen von Deutschland, Österreich und der Schweiz ein Städteraster (insgesamt 51 Städte, davon 40 in Deutschland) mitsamt Flussnetz aufweist usw. Die Frage, ob und inwieweit vor diesem Hintergrund ein Vergleich der jeweiligen Ergebnisse möglich ist, ist bislang unbeantwortet. Mehr noch: Die Frage, welche Kartierungsgrundlage in welcher Weise auf die Informanten wirkt und welche Art von Information mit den jeweiligen Grundkarten erhoben wird, ist bislang noch gar nicht gestellt worden. Damit ist der Gegenstand des vorliegenden Beitrages angesprochen. Im Zentrum unseres Untersuchungsinteresses steht dabei folgende Frage:

Lassen sich über einen Vergleich von Daten, die mit Grundkarten unterschiedlicher Informationsdichte und -qualität erhoben werden, Unterschiede in der Nennung und Verortung von Sprachräumen feststellen und inwieweit stehen solche Unterschiede - als Aktivierungsmuster mentaler Sprachraumrepräsentationen - mit den variierten Stimuli in Zusammenhang?

Aufbauend auf einer breit angelegten empirischen Untersuchung sollen im Folgenden außerdem verschiedene Ausprägungen mentaler Repräsentation von Sprachräumen offen gelegt werden. Unter einer mentalen Repräsentation von Sprachräumen verstehen wir dabei das sprecherseitig bewusst oder unbewusst vorliegende Wissen über regionale sprachliche Charakteristika und damit korrespondierende geographische Räume. ${ }^{3}$

Die Studie zielt damit nicht allein auf allgemeine und spezielle Konzeptualisierungen von Sprachräumen, sondern auch auf die Untersuchung interpretativer Eigenschaften sprachraumbezogener Mental Maps.

\section{$2 \quad$ Informanten und Datenerhebung}

Die Untersuchung fand im Dezember 2005 an einem Marburger Gymnasium statt. Als Probanden konnten 187 Schüler der 12. und 13. Jahrgangsstufe gewonnen werden. Indem wir uns auf den Besuch der Deutschkurse beschränkten, konnte sichergestellt werden, dass wir alle Schüler und zugleich jeden Schüler nur einmal erreichten. ${ }^{4}$ Damit basiert die Untersuchung auf der Erhebung von Daten einer definierten sozialen Gruppe, nämlich von Marburger Schülern im Alter zwischen 17 und 20 Jahren (Altersdurchschnitt: 18 Jahre). Von den insgesamt 187 Fragebögen wurden 24 für die weiteren Auswertungen nicht berück-

\footnotetext{
${ }^{3}$ Dass die mentalen Repräsentationen sprachgeographischer oder auch nur geographischer Räume nicht deckungsgleich mit deren konkreten Entsprechungen sind, sondern aufgrund der individuellen Wahrnehmung und kommunikativen Erfahrung subjektiv definiert werden, liegt auf der Hand. Gleichzeitig sind interindividuell unterschiedliche kommunikative Einflussfaktoren für das Entstehen und die Strukturierung mentaler Sprachräume anzunehmen (Medien, Wohnorte, Freunde ...), die die Ausbildung regionalsprachlicher Stereotype und Prototypen maßgeblich steuern.

${ }^{4}$ Die Autoren danken Henrike Hildebrandt für die Vermittlung der Informanten und die Organisation der Untersuchung vor Ort im Unterricht. Wir danken ebenfalls Herrn Jürgen-Udo Pfeiffer, dem Direktor der Martin-Luther-Schule, sowie den Lehrern der Deutschkurse, die der Durchführung des Tests zugestimmt und Teile ihrer Unterrichtszeit für uns freigestellt haben. Ein besonderer Dank gilt den beteiligten Schülern.
} 
sichtigt, da die primäre Sozialisation der betreffenden Schüler nicht in Deutschland stattfand. Es liegen uns somit insgesamt 163 Formulare zur Auswertung vor, darunter 89 Bögen weiblicher und 74 Bögen männlicher Informanten, die jeweils im Raum Marburg sozialisiert sind. ${ }^{5}$

An jeden Informanten wurde ein zweiseitiges Formular ausgegeben, auf dessen erster Seite eine spezifische Karte der Bundesrepublik Deutschland abgedruckt war (cf. Abb. 1). ${ }^{6}$ Zusätzlich wurde an die Informanten folgende mündliche Versuchsanweisung ausgegeben:

"Bitte tragen Sie auf der Karte (mit einem Kringel o. ä.) diejenigen Sprachräume ein, die ihnen bekannt sind!"7

Es wurde bewusst auf die Verwendung des Terminus Dialekt verzichtet. Damit sollte von vornherein eine begriffliche Einengung vermieden werden. Beabsichtigt war, jedes mögliche Konzept von Sprache - z. B. auch Gruppensprachen - verorten zu lassen. Vor allem aber sollte beobachtet werden können, ob und wie die Sprecher eine Verortung der Standardsprache ansetzen. Die strikte Vermeidung der Dialektreferenz in der Untersuchungsanleitung trägt außerdem dem Umstand Rechnung, dass die Alltagskategorien der Sprecher mit den wissenschaftlichen Begriffen nicht deckungsgleich sind. Vielmehr ist eine unscharfe kategorielle Trennung zwischen unterschiedlichen Sprechlagen im Bewusstsein der Sprecher anzunehmen: Regionale Akzente können ebenso als Dialekte empfunden und deklariert werden wie das lokale Platt. Das heißt mit anderen Worten: Da sich die mentalen Repräsentationen von Sprache über sprachliche Erfahrungen mit unterschiedlichen Stilen, Sprechlagen und Situationen strukturieren, sind sie mit exakten Sprechlagenabgrenzungen nicht grundsätzlich in Einklang zu bringen. Eine exakte Bestimmung der über die eingezeichneten Sprachräume repräsentierten Sprechlagen/Varietäten ist daher kaum möglich. Dies wird bei der Auswertung der Bögen zu berücksichtigen sein.

Insgesamt wurden an die Schüler sieben verschiedene Typen von Grundkarten ausgegeben. Diese Grundkartentypen wurden über alle Kurse alternativ ausgeteilt, d. h. jeder Nachbar hatte eine andere Grundkarte vor sich liegen. Damit war die Absicht verbunden, dass die Ergebnisse nicht gruppenspezifische, sondern informantenspezifische Geltung erreichen. Für jeden der sieben Grundkartentypen liegen zwischen 23 und 26 Beantwortungen vor. Bei der Auswahl bzw. der Erstellung dieser Grundkarten waren folgende Überlegungen maßgeblich: Bei der Verortung von Sprachraumwissen kommt es zur Überschneidung mehrerer Wissenskomponenten, wesentlich einer sprachbezogenen sowie einer geographischen. Diese geographische Komponente kann dabei ebenfalls durch unterschiedliche Erfahrungsaspekte geprägt sein. Sie kann sich auf Aspekte der geophysischen Wirklichkeit beziehen, kann aber auch von sozialgeographischen Aspekten geprägt sein, von politischen Bedingungen oder kulturellen Konzepten. Um nun das Sprachraumwissen der Informanten zu erschließen, müssen diese Bedingungen bei der Aufbereitung der Kartengrundlage berücksichtigt werden. Unsere Hypothese besteht darin, dass sich eine unterschiedliche Ausgestaltung der Grundkarte im Antwortverhalten der Informanten niederschlägt, Daraus leitet sich die übergeordnete Frage ab, ob das Erhebungsinstrument die Ergebnisse der Erhebung beeinflusst. Dabei können im Rahmen dieser Untersuchung keinesfalls alle möglichen Einflussfaktoren kontrolliert werden. Unsere Auswahl fiel daher auf einige wenige Informationstypen, die unterschiedliche soziokulturelle

\footnotetext{
${ }^{5}$ Es sei vorweg genommen, dass keine signifikanten Unterschiede zwischen männlichen und weiblichen Informanten festgestellt werden konnten. Die anschließende Analyse differenziert daher diesbezüglich nicht.

${ }^{6}$ Auf der zweiten Seite wurden relevante Sozialdaten (Alter, Geschlecht, Sozialisationsräume, Muttersprache etc.) und zusätzliche Informationen zu sprachraumbezogenem Wissen abgefragt.

7 Beim Ausfüllen der Fragebögen hatten die Schüler keine zeitlichen Vorgaben. Um eventuelle Beeinflussungen der Eintragungen auf den Karten zu vermeiden, wurde bei der Durchführung streng darauf geachtet, dass zuerst alle Eintragungen auf der Karte vorgenommen wurden, bevor die Seite mit den Zusatzfragen betrachtet wurde.
} 
wie geographische Aspekte der Strukturierung von Raum repräsentieren und die in den bislang vorliegenden Studien bereits verwendet wurden. Die schließlich verwendeten Grundkarten unterscheiden sich im Wesentlichen hinsichtlich der Informationsqualität und -dichte, vorrangig hinsichtlich der Angabe von Agglomerationspunkten (Städte unterschiedlicher Größe), politischen (Landesgrenzen) und topographischen Informationen (Flüsse, geophysisches Profil): ${ }^{8}$

1. Grundkarte: Staatsgrenze

2. Grundkarte: Staatsgrenze + Relief

3. Grundkarte: Staatsgrenze + Haupt- und Nebenflüsse

4. Grundkarte: Staatsgrenze + Bundesländer

5. Grundkarte: Staatsgrenze +15 Großstädte (> 800.000 Einwohner)

6. Grundkarte: Staatsgrenze +102 Städte

7. Grundkarte: Kombinationskarte (Staatsgrenze, Bundesländer, Städte, Flüsse) ${ }^{9}$
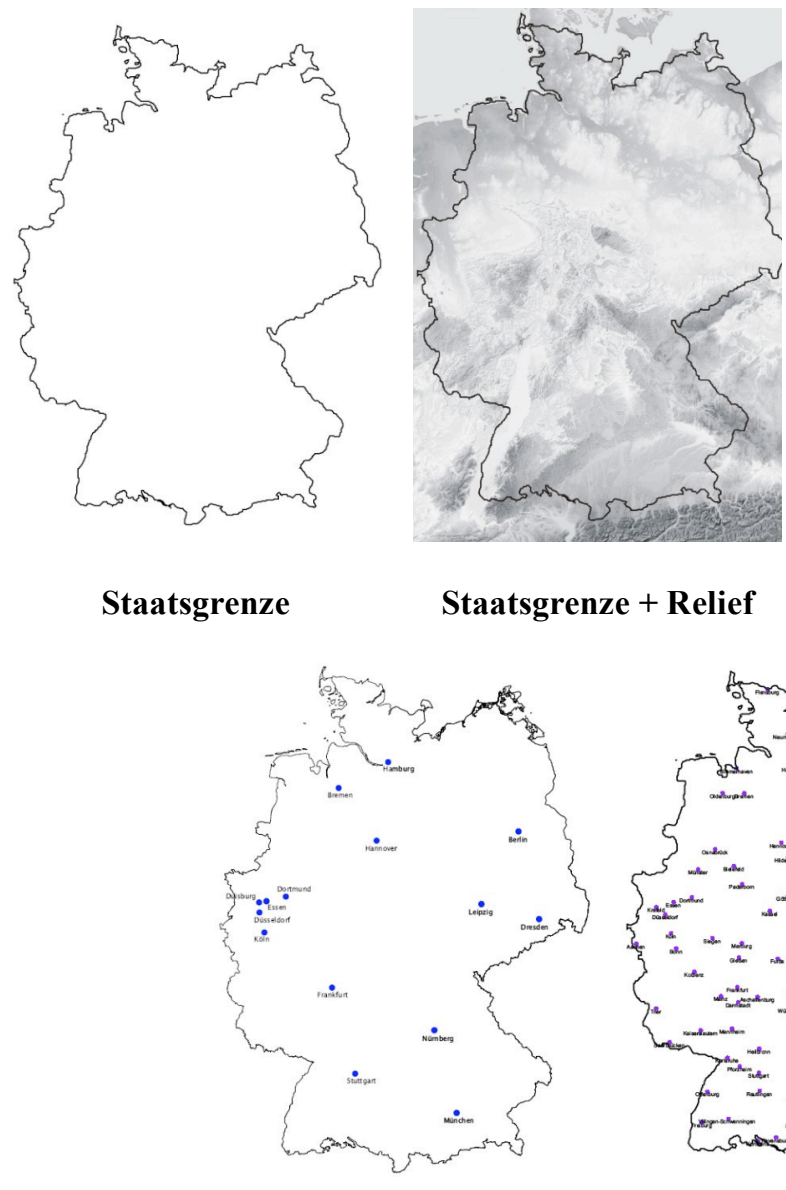

Staatsgrenze + Großstädte

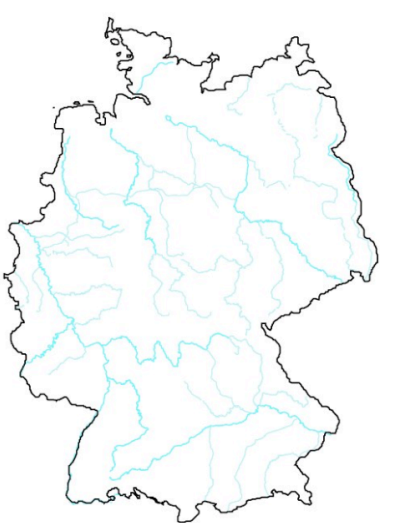

Staatsgrenze + Haupt- und Nebenflüsse

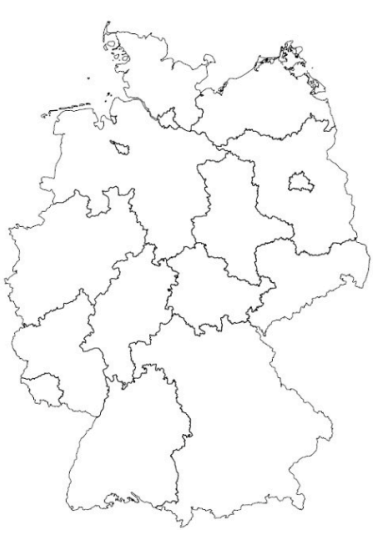

Staatsgrenze + Bundesländer
Abb. 1: Grundkartentypen bei der Erhebung

${ }^{8}$ Der Detailliertheitsgrad der Grundkarten wird dabei, ausgehend von der Staatsgrenze-Karte, wie folgt bestimmt: Staatsgrenze $<$ Flüsse $=$ Relief $<$ Bundesländer $<$ Großstädte $<$ Städte $<$ Kombination .

${ }^{9}$ Bei der Kombinationskarte wurde auf die zusätzliche Präsentation des Reliefs verzichtet, da die übrigen Informationen dann nicht mehr einwandfrei zu erkennen gewesen wären. Die Großstädte gehen im Städtenetz auf. Auf die Angabe von Verkehrswegen (Auer 2004) wurde verzichtet, da sie unserer Ansicht nach keinen wesentlichen Beitrag zur Strukturierung des sprachlichen Raums leisten, zumal bei der anzunehmenden geringen praktischen Erfahrung der Schüler als Kraftfahrer. Eine Teilkombination der Informationen untereinander - z. B. Flüsse und Städte auf einer Grundkarte - konnte unter Berücksichtung der Informantenanzahl nicht vorgenommen werden. 
Die übergeordnete Gemeinsamkeit der Grundkarten bildet die Darstellung der Staatsgrenze. Diese Angabe wurde als notwendig erachtet, um für spätere Auswertungen eine vergleichbare Bezugsgröße der Eintragungen sicherzustellen. Zudem wurden die Informanten damit auf das Gebiet der Bundesrepublik Deutschland fokussiert, das den eigentlichen Bezugsraum der Erhebungen darstellt. Damit einher geht natürlich eine methodische (und für den Informanten konzeptuelle) Verengung der Perspektive. Wird den Informanten dieser Bezugsrahmen allerdings nicht vorgegeben, so zeigen die Karten der Informanten deutlich verschiedene Arten der Strukturierung und Projektion mentaler Sprachräume, die teils auf sprachgeographische, teils auf sprachbiographische Motivationen zurückzuführen sind (cf. Hofer 2004). Ähnlich problematisch erscheint die Vermeidung einer klaren Versuchsanweisung, wie die Ergebnisse von Anders (2008) zeigen. ${ }^{10}$ Die notwendige methodisch-theoretische Implikation, nämlich die Trennung von Sprachwissen und geographischem Wissen, von mentaler Repräsentation und konkreter Erfahrungswelt, wird damit zwangsläufig vernachlässigt.

\section{$3 \quad$ Auswertung}

Grundlage der weiteren Analyse ist zunächst nur die Auswertung der ersten Formularseite mit den Sprachraumverortungen. In einem ersten Auswertungsschritt sollen anhand der Sprachraumnennungen diejenigen prominenten Sprachräume ermittelt werden, die unabhängig von den Grundkartentypen auf allen oder zumindest den meisten Karten aufzufinden sind. In einem zweiten Schritt soll dann die Anzahl der Eintragungen pro Grundkartentyp ermittelt und statistisch ausgewertet werden. Aus der zusätzlichen Analyse der Verortung prominenter Sprachräume auf den einzelnen Grundkarten sollen Hinweise auf wesentliche Unterschiede der Grundkartentypen gewonnen werden. Über die Auswertung ausgewählter Fragestellungen der zweiten Fragebogenseite schließlich wird versucht, die konzeptuelle Füllung der von den Informanten genannten Sprachräume wenigstens ansatzweise zu bestimmen.

\subsection{Sprachraumnennung}

\subsubsection{Allgemeines}

Insgesamt sind im Korpus 1.393 Sprachraumnennungen $\mathrm{zu}$ finden. Um ein bestimmtes Konzept zu konkretisieren, verwenden die Schüler dabei in der Regel relativ grobe "Label", die häufig an den Bundesländern angelehnt sind (z. B. Bayerisch) oder aber auf bestimmte Großlandschaften referieren (z. B. Norddeutsch). Wie noch zu zeigen sein wird, werden diese Konzepte nach Sprachraum und Grundkartentyp in deutlich unterschiedlicher Häufigkeit benannt. Eine eindeutige Koppelung bestimmter Typen von Nennungen an einen Karten- oder Informationstyp ist aus den Daten zunächst nicht abzuleiten. Besonders gut lässt sich dies an den seltenen Nennungen erkennen. So finden sich Städtenennungen (Hamburgisch, Frankfurterisch) auf allen Kartentypen, zumindest aber nicht ausschließlich auf den mit Städten versehenen Grundkarten. Ähnliches gilt für bundeslandbezogene Sprachraumnennungen, die

\footnotetext{
10 Anders (2008) unterscheidet mehrere unterschiedliche Kartierungs- und Kartentypen. Kartentypen beschreiben dabei in Ahnlehnung an Lynch (1960) Orientierungskategorien im geographischen wie mentalen Raum (etwa "Wege" "Grenzlinien", "Bereiche" oder "Brennpunkte", nach Anders die "formal-strukturelle Ebene"). Unter einem Kartierungstyp werden bei Anders die Ausfüllung und der Detailliertheitsgrad des Kartierungsbildes, die "inhaltlich-funktionale Ebene" gefasst ("exhaustiv", "selektiv", "autozentrisch"). Diese etwas unscharfen Kategorien werden im vorliegenden Fall durch die Vorgabe einer Versuchsanweisung auf einen relativ homogenen konzeptuellen Zugang verengt (im wesentlichen auf die Angabe von durch "Grenzlinien" umschlossene "Bereiche"), was zu strukturell anderen Ergebnissen führt (mentale Repräsentationen von Sprachräumen statt Repräsentationskategorien von Sprachwissen), aber den Vorteil der direkten Vergleichbarkeit bietet.
} 
nicht ausschließlich auf solchen Karten vorkommen, die auch die Bundesländergrenzen aufweisen. ${ }^{11}$

Als weitere Typen von Sprachraumnennungen finden sich z. B. im grenznahen Bereich des Kartierungsgebietes vereinzelte Hinweise auf fremdsprachliche Einflüsse (z. B. polnischer Einfluss, Dänisch, Französisch-Deutsch). Ebenso benennen die Schüler auf kleinere Gebiete bezogene Sprachräume, die häufig als Teile größerer Sprachräume angesehen und auch so bezeichnet werden (Tiefsächsisch, Unterbayerisch, Ostwestfälisch, Holsteinisch). Weiterhin kommen vereinzelt Scherzbenennungen von Räumen vor (Ossislang) sowie Fehlnennungen (Schwedisch (um Stuttgart, wohl statt Schwäbisch), Flämisch oder Holländisch (beides in Schleswig-Holstein)). Einen letzten Typ von Sprachraumnennungen bilden solche Räume, die sich auf individuelles Spezialwissen der Informanten beziehen und die zumeist in Verbindung mit der Herkunft des Informanten stehen (Kaiserslauterer Platt, Herborn, Marburger Plattdeutsch, Manisch (Gießen), Leetspeek (Thüringen)).

Für die weitere Auswertung der Daten wurde eine Klassifizierung der Nennungen vorgenommen. Erwartungsgemäß wird etwa ein Sprachraum Berlinerisch nicht immer in derselben Weise benannt. Im Korpus finden sich Alternativbenennungen wie Berlinerisch, Berliner Akzent, Berlinisch, Berliner Dialekt, Berliner, Berlinern, Berliner Schnauze (ike...), Berlin = icke etc. oder den aus Berlin mit icke. Solche Angaben wurden vorliegend zu einem Benennungstyp Berlinisch zusammengefasst. ${ }^{12}$ Die Klassifizierung aller Nennungen führt zum Beleg von insgesamt 96 unterschiedlichen Sprachräumen. Nach absoluten Häufigkeiten sortiert ergibt sich absteigend die folgende Reihung (Ausschnitt):

Bayerisch (155) $>$ Hessisch (139) $>$ Sächsisch (125) $>$ Schwäbisch (124) $>$ Berlinerisch (120) $>$ Norddeutsch (90) > Kölsch (82) > Hochdeutsch (59) > Ostdeutsch (42) > Friesisch (40) > Fränkisch (37) ...

Da die Nennungen nicht allein sprachliches Wissen reflektieren, sondern sich aus der Wechselwirkung von Sprachwissen und geographischem Wissen ergeben, sollen an dieser Stelle bereits die Antworten auf die den Informanten zusätzlich gestellte Frage nach weiteren Sprachräumen, die sie auf der Karte zwar nicht verorten konnten, aber dennoch kennen, angesprochen werden. Zwar verweist ein Teil der Informanten darauf, dass mehr als die eingezeichneten Sprachräume existieren müssten, konkrete Benennungen sind jedoch die Ausnahme. ${ }^{13}$

\subsubsection{Prominente Sprachräume}

Für die weitere Analyse wurden sämtliche Benennungstypen in eine Datenbank eingegeben und über eine hierarchische Clusteranalyse klassifiziert. ${ }^{14}$ In Beziehung gesetzt werden dabei die 96 Benennungen mit ihrem Vorkommen auf den einzelnen Grundkartentypen. Über die Analyse werden solche Sprachraumbenennungen ausgewiesen, die im Vergleich aller Grundkarten eine besonders große quantitative Ähnlichkeit zeigen. Daraus ergibt sich ein Hauptcluster, das insgesamt acht auf allen Grundkartentypen besonders frequente Fälle

\footnotetext{
${ }^{11}$ So findet sich die Nennung Baden-Württembergisch einzig auf der Grundkarte Städte, Saarländisch hingegen ist auf allen Grundkartentypen vertreten. Ebenso beziehen sich natürlich nicht sämtliche Nennungen auf der Grundkarte Bundesländer auf mit einem Bundesland assoziierte Sprachräume.

${ }^{12}$ Es soll herausgestellt werden, dass eine Diagliederung der Standard-Dialekt-Achse aus den Benennungen heraus nicht immer zu erkennen ist. Da es um die Benennung von Sprachräumen und nicht um die Benennung sprachstruktureller Differenzen geht, wurden mögliche vertikale Differenzierungen im Zweifel vernachlässigt. Die oben angeführten Sonderfälle (Scherzbenennungen, Fehlnennungen etc.) sind als unklassifizierte Einzelrepräsentationen in die Analyse eingegangen.

${ }^{13}$ Antworten dieser Art wären beispielsweise "Ja, gibt es bestimmt" oder "Viele, aber ich kann sie nicht benennen".

${ }^{14}$ Quadrierter euklidischer Abstand, Algorithmus nach Ward.
} 
enthält. Mit insgesamt 894 Nennungen machen diese acht Benennungstypen 64,18\% aller Nennungen aus. Zugleich wurden sie von jeweils mindestens einem Drittel der Informanten verzeichnet. Doch ist darüber hinaus eine weitergehende Differenzierung in Form zweier Subcluster zu erkennen. Dem ersten Subcluster gehören die Fälle Bayerisch, Berlinerisch, Sächsisch, Schwäbisch, Hessisch an, dem zweiten die Fälle Hochdeutsch, Kölsch und Norddeutsch.

$\begin{array}{ll}\text { 1. Subcluster } & \text { 2. Subcluster } \\ \text { Bayerisch } & \text { Hochdeutsch } \\ \text { Berlinerisch } & \text { Kölsch } \\ \text { Sächsisch } & \text { Norddeutsch } \\ \text { Schwäbisch } & \\ \text { Hessisch } & \end{array}$

Tab. 1: Subcluster 1 und 2 der Clusteranalyse $\mathrm{e}^{15}$

Diese Sprachräume lassen sich auf der Grundlage der Clusteranalyse als die prominenten Sprachräume des Korpus bezeichnen. Zugleich ist eine mehrgliedrige Hierarchie erkennbar. So sind etwa die Sprachräume des ersten Subclusters prominenter als diejenigen des zweiten, innerhalb des ersten Subclusters ist Bayerisch prominenter als Berlinerisch, Berlinerisch ist prominenter als Sächsisch usw. Umgekehrt lassen sich aus dieser Clusterung implikative Beziehungen in der Art ableiten, dass es bei den Informanten dieser Gruppe wahrscheinlich ist, dass z. B. eher Bayerisch als Hochdeutsch angegeben wird.

\subsubsection{Quantitativer Vergleich der Grundkartentypen}

Statistisch werden auf jedem Kartenblatt im Durchschnitt 8,5 Sprachräume eingezeichnet und benannt. Jedoch unterscheiden sich die Grundkartentypen untereinander teilweise deutlich. Die häufigsten Nennungen sind auf Grundkarte 7 festzustellen (Kombination; im Durchschnitt 9,58 Nennungen), die im Durchschnitt wenigsten Nennungen weist Grundkarte 2 (Relief) auf (7,35). Der Mittelwertsunterschied zwischen diesen beiden Kartentypen ist statistisch signifikant $(p=0,039) .{ }^{16}$ Dagegen sind die Mittelwerte der übrigen Kartentypen nicht signifikant verschieden.

Abb. 2 fasst dieses Ergebnis zusammen. Die Darstellung repräsentiert eine rein quantitative Auswertung der Testläufe pro Kartentyp. Es wurde hier die Anzahl aller benannten Sprachräume pro Kartenblatt summiert und anschließend für jeden Kartentyp das arithmetische Mittel ( $\overline{\mathrm{x}}$ ), die Standardabweichung (s) und die Spannweite (R) berechnet und eingetragen. Außerdem ist die Angabe zur höchsten und niedrigsten Anzahl der Nennungen pro Kartenblatt ( $\max$ vs. min) über einen horizontalen Strich markiert.

\footnotetext{
15 Die hier als prominent ausgewiesenen Sprachraumnennungen decken sich mit denen in anderen Studien, beispielsweise in der im Auftrag des Instituts für Deutsche Sprache 1997/98 durchgeführten bundesweiten Repräsentativerhebung (cf. Stickel/Volz 1999).

${ }^{16} t$-Test zur Prüfung unterschiedlicher Mittelwerte, zweiseitig, unabhängige Stichproben.
} 


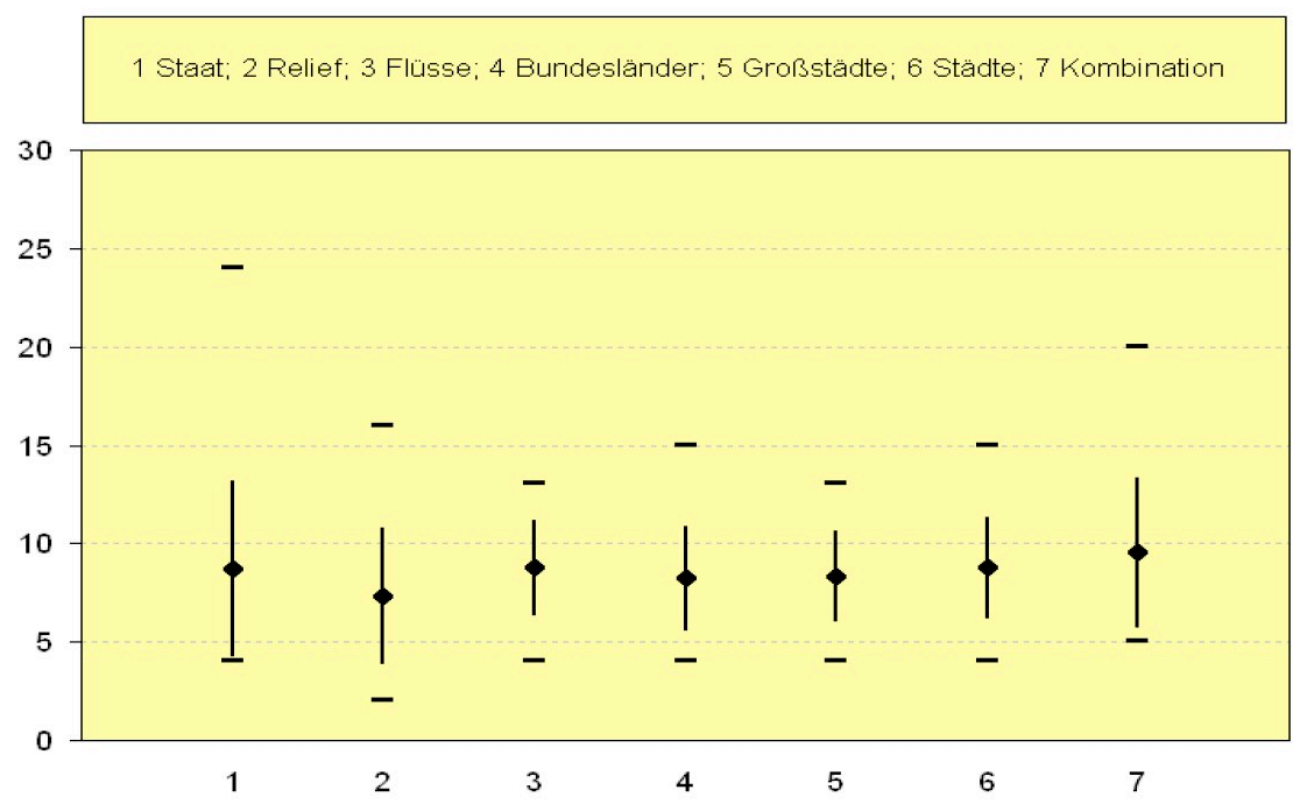

Abb. 2: Quantitative Variation der Sprachraumnennungen pro Kartentyp

\begin{tabular}{l|llllll} 
& $\mathrm{N}$ & $\overline{\mathrm{X}}$ & $\mathrm{s}$ & $\max$ & $\min$ & $\mathrm{R}$ \\
\hline Karte 1: Staatsgrenze & 24 & 8,75 & 4,41 & 24 & 4 & 20 \\
Karte 2: Relief & 23 & 7,35 & 3,42 & 16 & 2 & 14 \\
Karte 3: Flüsse & 25 & 8,76 & 2,39 & 13 & 4 & 9 \\
Karte 4: Bundesländer & 23 & 8,26 & 2,61 & 15 & 4 & 11 \\
Karte 5: Großstädte & 23 & 8,35 & 2,28 & 13 & 4 & 9 \\
Karte 6: Städte & 25 & 8,80 & 2,53 & 15 & 4 & 11 \\
Karte 7: Kombination & 26 & 9,58 & 3,76 & 20 & 5 & 15
\end{tabular}

Tab. 2: Statistische Häufigkeiten der Variation pro Kartentyp

Sehr viel aufschlussreicher als die Mittelwertsangabe ist der Vergleich der Variationsmaße, aus denen Hinweise auf die Heterogenität bzw. Homogenität der Nennungen abgeleitet werden können. Dies gilt insbesondere für die Standardabweichung (s) ${ }^{17}$, und zwar in folgendem Sinne: Je höher die Standardabweichung - d. h. die durchschnittliche Abweichung vom Mittelwert -, desto quantitativ heterogener sind die Antworten innerhalb eines Grundkartensamples. Je niedriger die Standardabweichung, desto quantitativ homogener sind die Antworten eines Grundkartensamples. Für die Spannweite (R) gilt in ähnlicher Weise: Je höher die Spannweite, desto heterogener sind die Antworten. Je geringer die Spannweite, desto homogener sind die Antworten.

Nach Ausweis von Abb. 2 sind vor allem zwei Grundkartentypen auffällig, nämlich die beiden Typen mit geringster und höchster Informationsdichte (1 Staatsgrenze vs. 7 Kombination). Diese Grundkarten belegen in der Benennung die höchste Variation. Dagegen variieren die Grundkartentypen 5 (Großstädte) und 3 (Flüsse) am geringsten. Weiteren Aufschluss über die Unterschiedlichkeit der Vorlagen bietet eine Signifikanzprüfung, die in Tab. 3 festgehalten ist. ${ }^{18}$

17 Die Standardabweichung wurde in die Graphik als Intervall [0+s; 0-s] eingetragen (vertikale Linie).

${ }^{18}$ F-Test zur Prüfung unterschiedlicher Varianzen, einseitig, unabhängige Stichproben. Signifikante Unterschiede in Fettdruck. 


\begin{tabular}{c|ccccccc} 
& 1 & 2 & 3 & 4 & 5 & 6 & 7 \\
\hline 1 & & & & & & \\
2 & 0,120 & & & & & & \\
3 & $\mathbf{0 , 0 0 2}$ & $\mathbf{0 , 0 4 4}$ & & & & & \\
4 & $\mathbf{0 , 0 0 8}$ & 0,105 & 0,333 & & & & \\
5 & $\mathbf{0 , 0 0 1}$ & $\mathbf{0 , 0 3 1}$ & 0,416 & 0,265 & & & \\
6 & $\mathbf{0 , 0 0 4}$ & 0,075 & 0,390 & 0,437 & 0,314 & & \\
7 & 0,218 & 0,332 & $\mathbf{0 , 0 1 5}$ & $\mathbf{0 , 0 4 4}$ & $\mathbf{0 , 0 1 0}$ & $\mathbf{0 , 0 2 8}$ &
\end{tabular}

Tab. 3: Signifikanz der Variation im Vergleich der Grundkartentypen (p-Werte)

Die Auswertung bestätigt den Eindruck aus Abb. 2. Die Kartentypen 1 Staatsgrenze und 7 Kombination sind signifikant von 3 Flüsse, 4 Bundesländer, 5 Großstädte und 6 Städte unterschieden. Untereinander besteht kein signifikanter Unterschied zwischen 1 und 7. Jedoch ist auch auffällig, dass Kartentyp 2 Relief von den Grundkarten Staatsgrenze und Kombination nicht unterschieden ist. Die größte Ähnlichkeit besteht also auf der Grundlage der Varianz zwischen den Grundkarten, die entweder nur die Staatsgrenze darstellen oder die Staatsgrenze zusammen mit dem Relief der Erdoberfläche oder aber, und das ist bemerkenswert, alle Informationen mit Ausnahme des Reliefs enthalten.

Das Ergebnis lässt sich folgendermaßen deuten: Unter Berücksichtigung des Umstands, dass Grundkarte 1 Staatsgrenze die höchsten Signifikanzwerte aufweist (etwa gegenüber Karte 5 Großstädte besteht ein Signifikanzniveau von 99,99\%), ist davon auszugehen, dass mit dieser Karte die geringste Beeinflussung der Informanten durch das Erhebungsinstrument vorliegt. Dies ist insofern ein erwartbares Ergebnis, als diese Karte mit der Staatsgrenze nur einen einzigen Stimulus enthält. Die hier eingetragenen Ergebnisse dürfen als Ausweis eines spontan verfügbaren Wissensbestands interpretiert werden. Demgegenüber ist auf den Grundkarten Bundesländer, Großstädte und Städte neben der Staatsgrenze jeweils ein weiterer Stimulus gesetzt. Dieser Stimulus scheint für die Informanten leitend zu sein, indem ihr Fokus auf einen bestimmten Aspekt des Raums und damit potenziell auch sprachlicher Räume gelenkt wird, nämlich zum Beispiel die Korrelation von Sprachräumen mit Stadtregionen oder Bundesländern (es zeigt sich also ein klarer Stimuluseffekt). Dies wird auch dadurch bestätigt, dass zwischen diesen drei Kartentypen kein signifikanter Unterschied besteht. Im Gegensatz zu Grundkarte 1 Staatsgrenze werden hier weitere Wissensbestände bzw. andere Wissensteilbestände aktiviert (cf. unten, Kap. 3.2.2).

Auf Grundkarte 7 (Kombination) ist die Heterogenität der Antworten ebenfalls sehr hoch. Allem Anschein nach wird hier durch die Fülle der Informationen bzw. die Anzahl der unterschiedlichen Stimuli der Fokus der Informanten nicht einseitig sondern variierend gesetzt. Auf diese Weise könnten unterschiedliche Wissensbestände aktiviert worden sein. Wie die Ergebnisse zeigen, führt eine Steigerung unterschiedlicher Stimuli rein quantitativ keineswegs zu einer Vereinheitlichung des Antwortschemas. Ganz im Gegenteil wird eine ähnliche Variationsbreite wie im Falle des Verzichts auf mehr als einen Stimulus erreicht. Zudem geht die Zunahme der Stimuli mit einer Zunahme der jeweiligen Eintragungen einher, denn immerhin hat dieser Kartentyp die im Durchschnitt häufigsten Nennungen von Sprachräumen. Es darf daraus geschlossen werden, dass diejenigen Informanten, die über ein individuell umfangreicheres Sprachraumwissen verfügen als andere, mit diesem Kartentyp in besonderem Maße die Möglichkeit gegeben finden, ihr Wissen zu aktivieren. Was das hinsichtlich der Qualität der Eintragungen, also der Raumbilder bedeutet, wird noch zu überprüfen sein. 
Abschließend sollen die Grundkartentypen Relief und Flüsse angesprochen werden, die offensichtlich Sonderfälle darstellen. Zwar ist auch bei der Relief-Karte eine auffällige Heterogenität der Beurteilung festzustellen. Da jedoch im Durchschnitt die wenigsten Regionen verzeichnet werden, kann angenommen werden, dass das geophysische Profil auf die Informanten irritierend wirkt. Der Grund hierfür könnte darin bestehen, dass mit der topographisch exakten Visualisierung - anders als bei der eher vagen Staatsgrenze-Karte - bei den Schülern der Anspruch geographischer Exaktheit steigt, so dass sie bei geographischer Unsicherheit eher dazu neigen, keine Angaben zu machen, anstatt vermeintliche Fehler zu produzieren. Grundsätzlich wäre Ähnliches auch für Karte 3 anzunehmen, auf der ein ausführliches Flussnetz verzeichnet ist. Allerdings ist das Gegenteil der Fall. Die Schüler verzeichnen vergleichsweise viele Sprachareale bei quantitativ vergleichsweise hoher Homogenität der Beurteilung.

\subsection{Sprachraumverortung}

Weil davon auszugehen ist, dass sich fast alle Sprachraumnennungen sowohl auf sprachliche als auch auf räumliche Wissenskomponenten beziehen, sollen in einem zweiten Analyseschritt - und anschließend an die Ergebnisse der statistischen Analyse - die eigentlichen Verortungen der benannten Sprachräume untersucht werden. Im Mittelpunkt steht dabei die Frage nach dem Zusammenhang der einzelnen Grundkartentypen und der Projektion von Sprachwissensinhalten in den geographischen bzw. topologischen Raum.

\subsubsection{Gesamtüberblick}

Die weiteren Ausführungen konzentrieren sich auf diejenigen acht Nennungen, die in der voran stehenden statistischen Analyse als die prominenten Sprachräume des Korpus ermittelt wurden. Es soll gefragt werden, inwieweit im vorliegenden Fall überhaupt vergleichbare Lokalisationen der typischen Sprachräume erfolgt sind und zweitens, ob sich neben den bereits erbrachten quantitativen Evidenzen auch in der Analyse der Sprachraumlokalisationen Hinweise auf die Charakteristik der Grundkartentypen ableiten lassen.

Die wesentliche Information im Zusammenhang mit der Verortung der Nennungen ist der Deckungsgrad der eingezeichneten Areale. Aus der Überschneidung der einzelnen Eintragungen pro Sprachraumnennung ergibt sich eine Schnittmenge, an der je nach Kartentyp und Sprachraum unterschiedlich viele Eintragungen beteiligt sein können. Diese Schnittmenge lässt sich in zweierlei Hinsicht definieren. Erstens über ihre Größe, d. h. hinsichtlich ihrer Ausdehnung auf dem Kartenblatt, sowie zweitens über ihre Dichte, d. h. hinsichtlich der Anzahl der an der Schnittmenge beteiligten Eintragungen. Es ist ferner davon auszugehen, dass je nach Grundkartentyp unterschiedliche Schnittmengen entstehen können. Um die Eintragungen direkt vergleichen $\mathrm{zu}$ können, wurden in einem ersten Schritt sämtliche Eintragungen digitalisiert und in Polygone gewandelt. ${ }^{19}$ Die monochromen Grafiken in Abb. 3 zeigen auf dieser Grundlage die prominenten Sprachräume der Clusteranalyse, deren Einzelverortungen als einzelne Objekte definiert und in Form kreuzschraffierter Layer mit konstantem Linienabstand und konstanter Linienbreite undifferenziert übereinander gelegt wurden. ${ }^{20}$ Dies führt zu einer Überlagerung der Schraffuren, die dort dichter wird, wo sich mehrere Räume überschneiden. Die dunklen Schnittmengen bilden die Kernräume oder Lokalisationskerne der eingezeichneten Gesamtflächen für eine Sprachraumnennung, die helleren Gebiete bezeichnen wir als Grenzgebiete oder Teildeckungsgebiete. Die einzelnen Sprachraumverortungen treten so als mehr oder weniger konsistente Flächen in Erscheinung.

${ }^{19}$ An dieser Stelle sei für die Mitarbeit an den Digitalisierungen herzlich gedankt: Sybille Felix, Miriam Hildenbrand, Inga Passow, Mareike Sachau, Dorothee Weissenfels und Constanze Wellendorf.

${ }^{20}$ Es handelt sich um sämtliche Eintragungen aller Grundkartentypen. 
Dieser Flächencharakter der Sprachräume in der Kombination aller Nennungen ist zu betonen, denn es fällt auf, dass - unabhängig von der Grundkarte - stets zusammenhängende Flächen identifizierbar sind, die in einer bestimmten Teilregion der Karte verortet sind. Unterschiede betreffen neben der Verortung auf der Karte erstens die Gesamtgröße der jeweiligen Variationsfläche, zweitens das Vorhandensein eines einzelnen Kernraumes im Sinne einer erkennbaren Schnittmenge aller bzw. der meisten Eintragungen, drittens Lage und Größe des Lokalisationskerns sowie viertens Lage und Größe des Teildeckungsgebietes um den Lokalisationskern herum.

Darüber hinaus wurden die Lokalisationskerne einer weiterführenden quantitativen Analyse unterzogen, deren Ergebnis in Abb. 3 durch farbige Grafiken dargestellt ist. Diese Grafiken zeigen die Struktur der einzelnen Lokalisationskerne in Form einer kumulativen Hierarchisierung. Es wurde eine Klassenbildung in 10\%-Schritten auf der Grundlage identischer Distanzen $^{21}$ gewählt. Damit liegt ein für die Interpretation der Daten sinnvoll nutzbares Maß vor. ${ }^{22}$ Die bisherigen Ausführungen haben bereits verdeutlicht, dass diese polychromen Grafiken auf die Deckungsgrade der Eintragungen hinweisen. Ein Deckungsgrad von z. B. 61-70\% besagt, dass sich 61-70\% der Eintragungen im betreffenden Areal überschneiden. Die hier einfarbig ausgewiesenen Schnittmengen sind also an die Deckungsdichte aller Einragungen für einen Benennungstyp geknüpft. Typischerweise ist das zentrale Areal dasjenige mit der höchsten Überschneidungsrate. Je höher der Prozentwert dieses Kerns ist, desto ähnlicher sind die Verortungen der Informanten insgesamt. ${ }^{23}$ Am ähnlichsten ist demgemäß die Verortung im Falle Bayerisch (81-90\%), sodann bei Hessisch (71-80\%) und Sächsisch (61-70\%). Besonders heterogen sind demgegenüber die Verortungen von Hochdeutsch, Berlinerisch und Kölsch (je 31-40\%).

21 Tests mit anderen Klassifizierungen, z. B. dem Algorithmus nach Jenks (natural breaks), wurden durchgeführt, die Ergebnisse unterschieden sich im vorliegenden Fall hinsichtlich der Klassenbildung nur geringfügig. Cf. hierzu auch die technischen Vorgehensweisen von Montgomery (2007) sowie Long/Onishi (o. J.).

22 Versuche mit anderen Klassenbildungen führten in der Regel zu nur eingeschränkt brauchbaren Ergebnissen.

${ }^{23}$ Die Ausdehnung des Zentrums ist als Beurteilungskriterium problematisch, weil die verorteten Konzepte realiter eine unterschiedliche Ausdehnung haben, cf. z. B. Kölsch vs. Bayerisch. Die Ausdehnung des Zentrums wird hier daher nicht weiter verfolgt. 


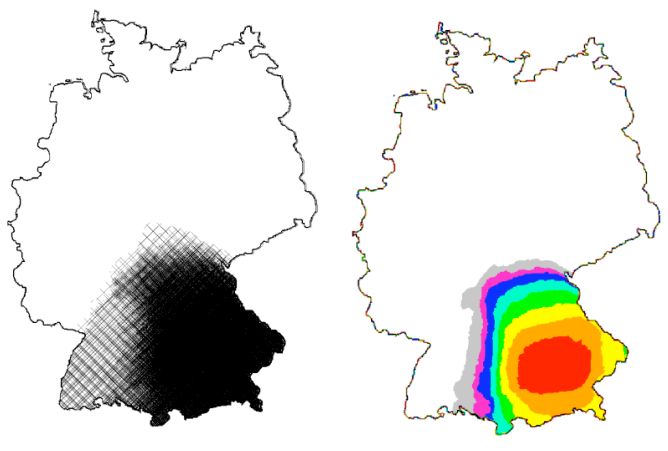

Bayerisch
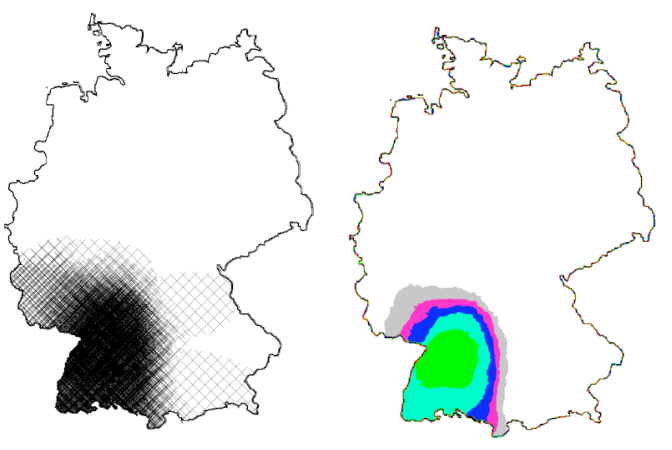

Schwäbisch
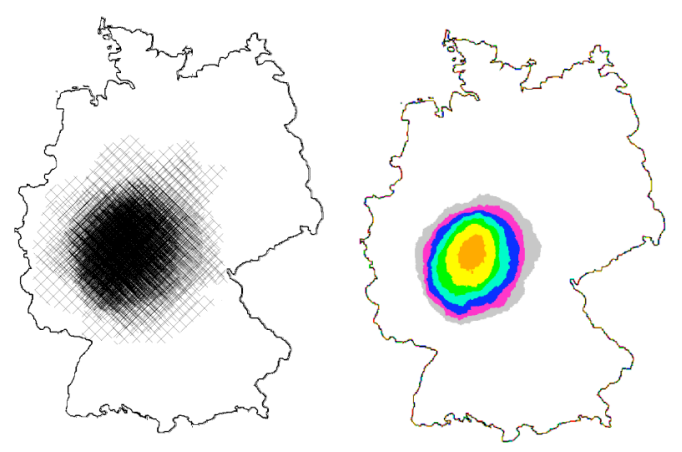

Hessisch
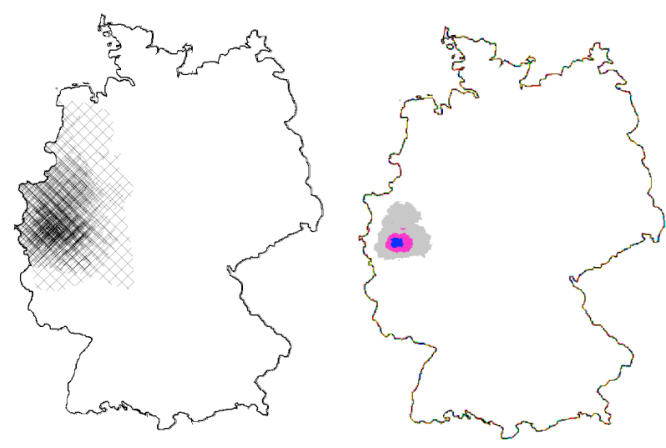

Kölsch
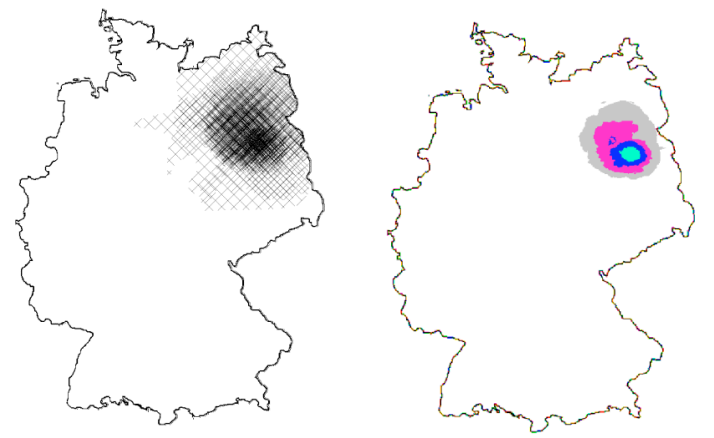

cumulation

percentage

- $91-100$

$81-90$

$71-80$

$61-70$

$51-60$

$41-50$

$31-40$

$21-30$

$11-20$

0 - 10

Berlinerisch

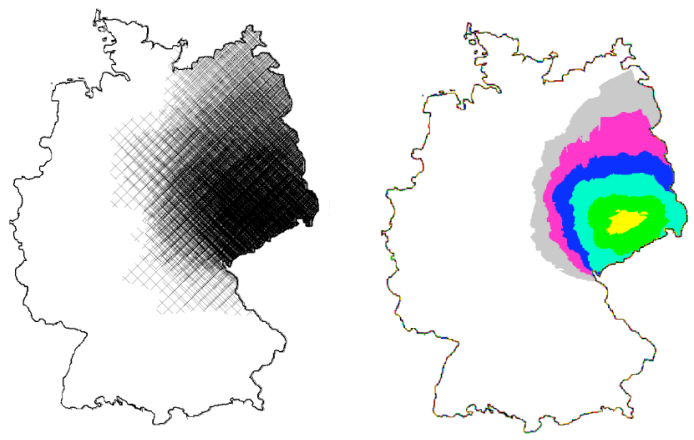

Sächsisch
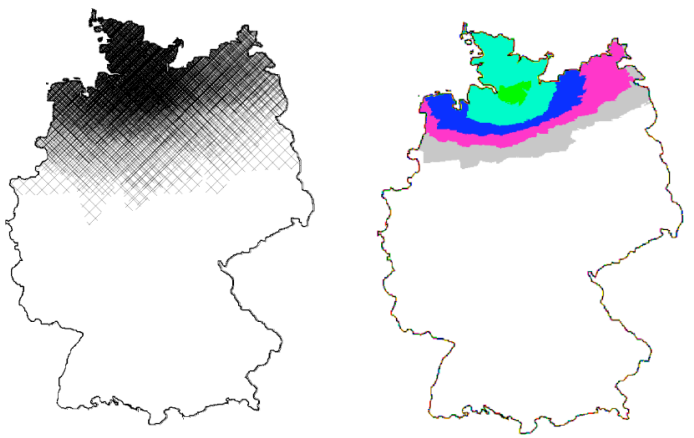

Norddeutsch
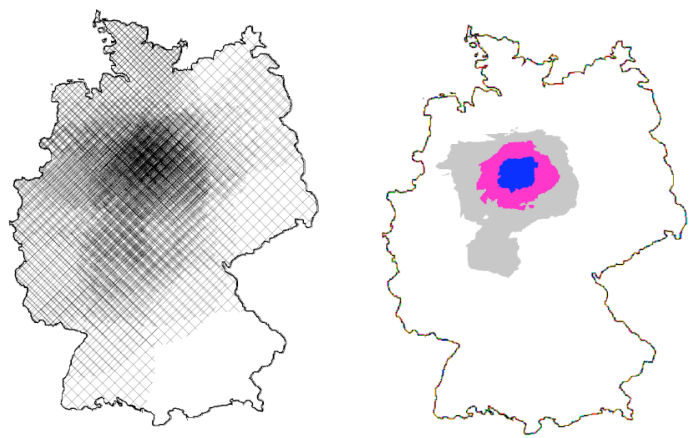

Hochdeutsch

Abb. 3: Kumulative Verortung der acht signifikanten Sprachraumnennungen 
Analog zur Hierarchisierung der Sprachraumbenennungen lässt sich darauf aufbauend nun auch eine Hierarchisierung der Sprachraumverortungen aufstellen, die hier in absteigender Anordnung nach den Prozentwerten der Kerndichte angegeben ist:

\begin{tabular}{lll} 
Sprachraum & Dichte Kern & Ausdehnung Kern ${ }^{24}$ \\
\hline 1. Bayerisch & $81-90 \%$ & $12,6 \%$ \\
2. Hessisch & $71-80 \%$ & $2,5 \%$ \\
3. Sächsisch & $61-70 \%$ & $1,7 \%$ \\
4. Schwäbisch & $51-60 \%$ & $10,0 \%$ \\
5. Norddeutsch & $51-60 \%$ & $1,6 \%$ \\
6. Hochdeutsch & $31-40 \%$ & $1,5 \%$ \\
7. Berlinerisch & $31-40 \%$ & $1,2 \%$ \\
8. Kölsch & $31-40 \%$ & $0,65 \%$
\end{tabular}

Tab. 4: Dichte- und Flächenrelationen

Diese Hierarchie ist mindestens hinsichtlich der ersten vier Positionen deckungsgleich mit der Häufigkeit der Sprachraumbenennungen (cf. Kap. 3.1.1). Die Häufigkeit der Sprachraumbenennung korreliert somit in diesen Fällen mit der konsistenten Verortung des spezifischen Konzepts. Darüber hinaus ist in die Tabelle der prozentuale Anteil der Kernfläche an der Gesamtfläche aller Eintragungen aufgenommen. Der Wert beziffert etwa für das Sächsische den Gesamtanteil des gelben Kerns (Abb. 3) an der in der monochromen Darstellung sichtbaren Gesamtfläche. Eine Korrelation zwischen der Größe des Kerns und der Größe der Gesamtfläche besteht nicht. Das bedeutet mit anderen Worten, dass die Fläche der Überschneidungen - anders als ihre Dichte - nicht mit der Häufigkeit der Sprachraumbenennungen in Zusammenhang zu bringen ist.

Auffällig sind diesbezüglich v. a. die Werte für das Bayerische und das Schwäbische. In diesen Fällen liegen die mit Abstand höchsten Werte vor. Ausschlaggebend für diese hohen Werte dürfte nicht zuletzt die geometrische Gestalt des Raumkörpers Staatsgrenze sein, der die Markierung in diesem Abschnitt wesentlich leitet (cf. Kap. 3.2.2.1). Die Verortung der Sprachräume erfolgte hier mit größtmöglicher Übereinstimmung. An den ins Binnenland gerichteten Rändern des Kerns befindet sich jeweils ein dichtes Teildeckungsgebiet, das aber zum einen auch aus überlagernden Schraffuren besteht und das zum anderen, gemessen am Kernraum, relativ schmal ausfällt. Relativ hoch ist diesbezüglich noch die Ausdehnung im Fall des Hessischen - und damit in der Untersuchungsregion -, was für eine relative Konsistenz der Eintragungen spricht.

Etwas anders verhält es sich bei der Eintragung Sächsisch. Obwohl auch in diesem Fall eine relativ hohe Dichte des Kerns gegeben ist, ist seine Ausdehnung eher durchschnittlich. Das Grenzgebiet fällt gegenüber den bereits erwähnten Sprachräumen weitläufiger aus. Die Zuordnungen der Informanten waren also weniger einheitlich. Ähnlich ist auch das Ergebnis im Falle des norddeutschen Areals, wobei allerdings hier die Kerndichte geringer ist.

Die auf die Städte Berlin und Köln bezogenen Sprachraumnennungen Berlinerisch und Kölsch sind hinsichtlich ihrer Gesamtflächen von geringerer Größe, die Kernräume sind besonders klein ausgeprägt. Besonders Kölsch bleibt vage. Dies kommt dadurch zustande, dass auch die Einzeleintragungen der Informanten deutlich kleinräumiger sind als bei den anderen Sprachräumen und gleichzeitig bei einzelnen Grundkartentypen eine starke Unsicherheit hinsichtlich der geographisch exakten Lage zu beobachten ist. $\mathrm{Zu}$ bemerken ist, dass Berlin auf vier Grundkarten bereits als Information vorgegeben ist (Bundesländer, Groß-

\footnotetext{
${ }^{24}$ Prozentual zur Gesamtfläche der Eintragungen.
} 
städte, Städte und Kombination), während Köln nur auf den drei Karten mit StädteInformationen als Orientierungsgröße enthalten ist.

Einen Sonderfall bildet der Sprachraum Hochdeutsch, und zwar insofern, als die Informanten insgesamt in fast allen Bereichen des Kartenblattes Eintragungen vorgenommen haben. Dennoch ist ein Kernraum von relativ geringer Dichte in zentraler Lage erkennbar, ein weiterer, noch schwächer ausgeprägter Kernraum schließt sich unmittelbar darunter an. Von besonderer Bedeutung ist jedoch ein anderer Umstand: Ausgerechnet jener Abschnitt der Grundkarte ist von Verortungen ausgespart, in dem typischerweise der bayerische Sprachraum lokalisiert ist. Es sei daran erinnert, dass Bayerisch in der Clusteranalyse als der prominenteste Sprachraum überhaupt belegt wurde und dieser Sprachraum darüber hinaus mit größter Konsistenz verortet wird. Darauf aufbauend lässt sich das Hochdeutsche nach den Verortungen der Informanten über eine Negativdefinition bestimmen: "Hochdeutsch ist das, was nicht Bayerisch ist". Das Bayerische bildet so gesehen einen (konzeptuellen) Gegenpol zum Hochdeutschen. Ähnliches zeigt sich für den Abschnitt des Kartenblattes, der mit dem Bundesland Sachsen korreliert, insofern, als dieser lediglich von einem Informanten dem Hochdeutschen zugerechnet wird. Dies deutet bereits an, dass ein Kriterium "Dialekttiefe" im Urteil der Informanten eher nachrangig ist. ${ }^{25}$

Damit kann vorerst festgehalten werden, dass die ausgewiesenen Nennungen des Korpus grundsätzlich vergleichbar lokalisiert werden. In einzelnen - prominenten - Fällen zeigt sich eine hohe Ähnlichkeit zwischen der Häufigkeit der Nennung und der Verortung einzelner Sprachraumkonzepte. Darüber hinaus ergeben sich Hinweise auf die Abhängigkeit der Verortungen von der geometrischen Gestalt des vorgegebenen Raumkörpers (Staatsgrenze). Damit fällt der Blick auf die aus den einzelnen Grundkartentypen ableitbaren Stimuluseffekte.

\subsubsection{Grundkartentypen}

Im Anschluss an die Auswertung der Sprachraumnennungen soll im Folgenden untersucht werden, ob die über die statistische Auswertung ermittelten Effekte der einzelnen Grundkarten auch in der Verortung der genannten Sprachräume ihre Entsprechung finden. In die Analyse der Verortung werden diejenigen Sprachräume einbezogen, die durch eine geschlossene räumliche Markierung eindeutig einem bestimmten Areal zugewiesen werden. ${ }^{26}$ Daran anschließend definieren wir einen Sprachraum dann als für einen bestimmten Grundkartentyp prominent, wenn er auf mindestens einem Drittel der vorliegenden Bögen für diesen Kartentyp eindeutig verortet ist (Drittelkriterium). Dieses Kriterium ist aus der Clusterung des Gesamtsamples abgeleitet: Die von den übrigen Nennungen signifikant unterschiedenen Sprachraumnennungen der ersten beiden Subcluster wurden jeweils von mindestens einem Drittel der Informanten verzeichnet. ${ }^{27}$

\subsubsection{Grundkarte Staatsgrenze}

Den Ausgangspunkt für die Analyse stellt die Grundkarte Staatsgrenze dar. Abgesehen von der auf allen Karten vorgegebenen Bundesaußengrenze wird hier keinerlei weitere Raum-

\footnotetext{
${ }^{25}$ In der Konsequenz sind eher stereotype bzw. prototypische Bewertungen regionaler Sprache als Strukturierungsfaktoren für den sprachlichen Raum anzunehmen. Cf. unten, Kap. 3.3 und Kap. 5.

${ }^{26}$ Als geschlossen gilt eine Markierung, die entweder in sich abgeschlossen ist (z. B. ein Oval) oder deren Begrenzung durch angrenzende Sprachräume bzw. die Bundesaußengrenze eindeutig definiert ist. Sprachraumnennungen, die ohne eine exakte räumliche Markierung in die Karte eingetragen wurden (beispielsweise lediglich unterstrichene Städtenamen), werden im Folgenden nicht berücksichtigt. Es sei zugleich herausgestellt, dass solche Eintragungen aufgrund der Fragestellung, die eine geschlossene Markierung forderte, äußerst selten vorkommen.

${ }^{27}$ Cf. Kap. 3.1.2. Die statistische Auswertung (Clusteranalyse) der einzelnen Kartentypen führt zu keinen interpretierbaren Ergebnissen.
} 
information geboten, die die Verortung der Sprachräume beeinflussen könnte. Diese politische Information dient dem Betrachter als räumlicher Orientierungsrahmen, von dem aus der anzugebende Sprachraum erschlossen werden kann. Das Drittelkriterium weist, bei 24 ausgewerteten Fragebögen, für diesen Grundkartentyp die folgenden Sprachräume als prominent aus:

\section{Staatsgrenze $(\mathrm{N}=24)$ :}

Bayerisch (24) > Hessisch (22) > Schwäbisch (21) > Sächsisch (18) > Berlinerisch (15) > Norddeutsch (13) $>$ Friesisch (8), Kölsch (8). ${ }^{28}$

Auffällig ist dabei zunächst, dass es sich, mit Ausnahme des Friesischen, um dieselben Räume handelt, die auch nach Ausweis der Clusteranalyse charakteristisch für das Gesamtsample sind. Dafür entfällt das im Bezug auf das Gesamtsample prominente Hochdeutsch. Für die Verortung dieser Räume ist offensichtlich, dass die Informanten sich an der geometrischen Gestalt des vorgegebenen "Raumkörpers" orientieren und darauf aufbauend eine individuell unterschiedlich ausgeprägte Topologie der Sprachräume entwerfen. Leitend ist dabei das geographische Wissen der Informanten, das in Relation zum vorgegebenen Grenzverlauf gesetzt wird. Dieser Grenzverlauf gibt dem Betrachter mögliche Ansatzpunkte vor, welche für die genauere, geometrisch orientierte Verortung genutzt werden. Als solche markante Ansatzpunkte dienen vorrangig auffällige Ein- oder Ausbuchtungen des Grenzverlaufs.

Dieses Vorgehen hat sich bereits im Rahmen der Berechnung der Kerndichte angedeutet (Kap. 3.2.1). Die durch das geographische Wissen gesteuerte Orientierung an der geometrischen Form des Kartierungsgebiets scheint etwa im Falle von Schwäbisch und Bayerisch besonders gut zu gelingen, und zwar deshalb, weil sich für das Gebiet der Bundesrepublik Lage und Erstreckung der geographischen Räume wie der korrespondierenden subjektiven Sprachräume relativ leicht anhand der geometrischen Form der Bundesaußengrenzen erschließen lassen. ${ }^{29}$ Für den Fall Bayerisch könnte eine Orientierung etwa wie in Abb. 4 dargestellt ausgesehen haben: Der Betrachter halbiert das Kartenbild zunächst in der Vertikalen und setzt die Nennung Bayerisch schließlich in den rechten unteren Teil des Bildes, und zwar unterhalb einer gedachten Verknüpfung zweier markanter Seitenpunkte. ${ }^{30}$ Im Falle Hessisch wird ebenfalls in der Vertikalen halbiert. Die weitere Orientierung der Informanten scheint auf die linke Seite zu fallen, wo sich ebenfalls markante Grenzpunkte erkennen lassen, die auf die weitere Verortung beeinflussend gewirkt haben mögen. Norddeutsch dagegen scheint von den Informanten gleich gesetzt zu werden mit "Sprache an der Küste" bzw. "Sprache des Nordens". ${ }^{31}$ Die Eintragungen in die anderen unten dargestellten Kartentypen lassen sich in ähnlicher Weise erschließen.

Es lässt sich für jeden der unten in Auswahl dargestellten prominenten Sprachräume eine Verortungsstrategie nachvollziehen. Diese Verortungsstrategien sind als Rekonstruktionsroutinen für das individuelle geographische Wissen $\mathrm{zu}$ verstehen, das in die Kartierungsgrundlage eingepasst werden muss. Da allerdings das geographische Wissen der Informanten

\footnotetext{
${ }^{28}$ Die in Klammern angegebenen Zahlen beziehen sich auf die Vorkommenshäufigkeit der einzelnen Verortungen.

${ }^{29}$ Die relative Übereinstimmung dieser beiden Projektionskategorien lässt sich dabei aus den besonderen historischen Bedingungen der Entwicklung des deutschen Sprachraums wie Deutschlands ableiten.

30 Sofern differenziert, repräsentiert die Art der Linien eine mögliche Reihenfolge der Erschließung: Strichlinie = erste Orientierung; Punktlinie = weiter führende Lokalisierung. Die hier vorgeführte Erschließungsstrategie ist eine von mehreren individuell möglichen Verfahrensweisen. Ebenso sind z. B. Strategien zu erwägen, die auf einer rechtwinkligen Gliederung des Raumkörpers aufbauen o.ä.

${ }^{31}$ Zur konzeptuellen Überlagerung der Räume Norddeutsch und Friesisch, die sich in der weitgehend deckungsgleichen Verortung der beiden Nennungen niederschlägt, cf. Kap 5.
} 
individuell höchst unterschiedlich ausfällt, kommt es auf diesem Grundkartentyp zu einer extrem hohen Variation in der Anzahl der eingetragenen Nennungen (cf. Kap. 3.2.1). Die Spannweite ist - wie erwähnt - in diesem Fall so hoch wie bei sonst keinem anderen Grundkartentyp (4-24 Nennungen). Die Eintragungen zeigen aber auch, dass durch die Vorgabe des Grenzverlaufs ein Stimulus gesetzt ist, der zu eindeutigen und interpretierbaren Ergebnissen führt.

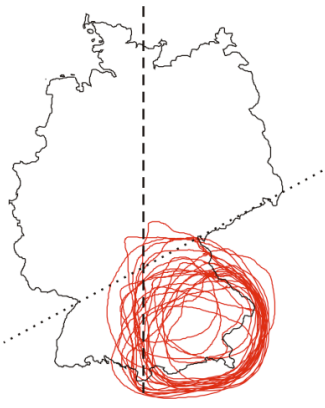

Bayerisch

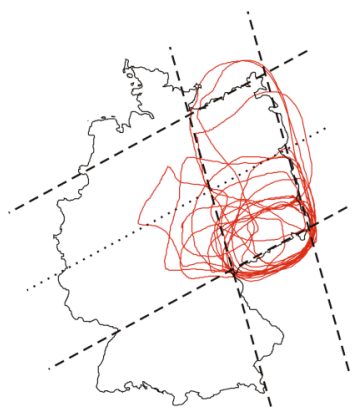

Sächsisch

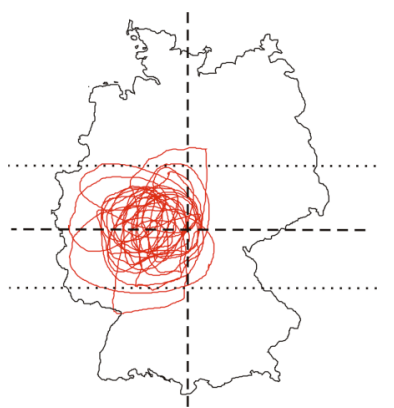

Hessisch

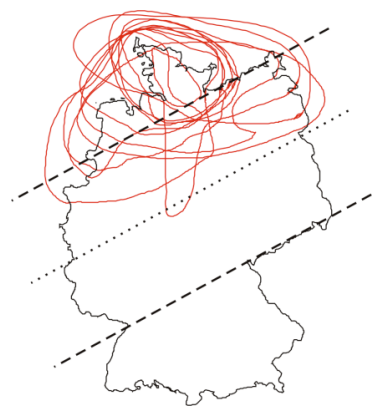

Norddeutsch

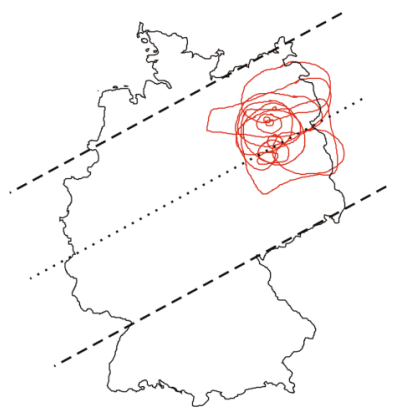

Berlinerisch

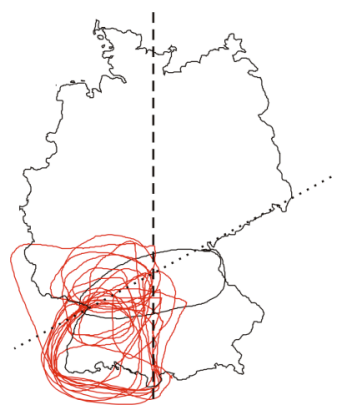

Schwäbisch

Abb. 4: Beispiele möglicher Verortungsstrategien für die Grundkarte Staatsgrenze

Gerade weil die Informationsdichte für den Grundkartentyp Staatsgrenze - abgesehen von der Primärstrukturierung des Kartierungsraumes durch die Bundesaußengrenzen - minimal ist und keine zusätzlichen Stimuli als Lenkungsfaktoren für die Kartierung dargeboten werden, können die vorgenommenen Eintragungen als maximal spontaner Wissensbestand interpretiert werden.

Es kann somit festgehalten werden: Die Informanten realisieren beim Grundkartentyp Staatsgrenze grundsätzlich ähnliche Lokalisationen. Es lassen sich Verortungsstrategien annehmen, die dafür sprechen, dass die Schüler immerhin eine so ausgeprägte geographische Kenntnis haben, dass die Angaben in relativem Einklang stehen. Auch wenn die Informanten sich dabei an geometrischen Besonderheiten der vorgegebenen Bundesaußengrenze orientieren mögen, ist doch auffällig, dass eben diese Orientierungsmuster sich untereinander stark ähneln und damit den Schluss auf eine weitgehend übereinstimmende Vorstellung von der regionalen Verortung der Sprachräume auf Seiten der Informanten zulassen. ${ }^{32}$ Gleichzeitig reflektieren diese Orientierungsmuster das Wissen der Informanten um die tatsächliche geographische Lage der benannten Räume, das anhand der geometrischen Charakteristika des Kartierungsgebietes reproduziert und strukturiert werden kann. Es wird im Folgenden zu zeigen sein, inwiefern sich durch die variierende Ausgangsinformation auf den Grundkarten diese Verortungsstrategien verändern und ob sich diese Veränderungen mit der Informationsdichte und -qualität der jeweiligen Grundkarte in Zusammenhang bringen lassen.

\footnotetext{
32 Nebenbei soll erwähnt werden, dass die Schüler damit alle Erwartungen ihrer Lehrer ins Gegenteil verkehren.
} 


\subsubsection{Grundkarten Relief und Flüsse}

Relief $(\mathrm{N}=23)$ :

Hessisch (18) > Sächsisch (17) > Bayerisch (13), Berlinerisch (13), Norddeutsch (13), Schwäbisch (13) $>$ Hochdeutsch (8)

Flüsse $(\mathrm{N}=25)$ :

Bayerisch (25), Hessisch (22), Sächsisch (21), Berlinerisch (20), Schwäbisch (20), Kölsch (14), Norddeutsch (14), Hochdeutsch (9)

Für den nächsten Analyseschritt bieten sich nun die beiden Grundkarten an, auf denen neben der Bundesaußengrenze lediglich eine weitere raumgeographische Information gegeben ist: die Karten Flüsse und Relief. Betrachtet man die auf dem jeweiligen Kartenblatt eingetragenen Positionen der Sprachräume, so entsprechen die Verortungen beider Grundkartentypen weitgehend denjenigen der Staatsgrenze-Karte. In quantitativer Hinsicht weisen die Kartentypen nach Anwendung des Drittelkriteriums mit jeweils einer Ausnahme dieselben Sprachräume als prominent aus, die auch auf der zuvor betrachteten StaatsgrenzeKarte repräsentiert sind (Abb. 4). Im Falle der Karte Flüsse stimmen die prominenten Sprachräume exakt mit denen des Gesamtsamples überein (Abb. 3).

Auffällig ist jedoch, dass die Verortungshäufigkeiten auf der Relief-Karte deutlich unter denen der anderen Karten liegen. Für das Bayerische etwa weist die Karte Relief lediglich halb so viele Eintragungen auf wie die Flüsse-Karte. Für alle anderen Sprachräume dieses Kartentyps gilt dies in weniger drastischem Ausmaß ebenso. Darüber hinaus liegt mit der Relief-Karte der einzige Grundkartentyp vor, auf dem der Sprachraum Kölsch nicht signifikant ist. Es kann also davon ausgegangen werden, dass die geophysische Information des Reliefs die Eintragungen der Schüler insofern beeinflusst, als durch das Relief das Untersuchungsgebiet zwar deutlich strukturiert wird, die gegebene Information aber nicht konkret genug ist, um eine räumliche Orientierung zu leisten, sondern diese, im Vergleich zu der Karte Staatsgrenze, sogar eher behindert, so dass die Schüler deutlich weniger Eintragungen vornehmen als auf den anderen Karten. ${ }^{33}$

Demgegenüber stellt das Flussnetz für die Schüler in Bezug auf die statistisch signifikanten Sprachräume im Normalfall keine hindernde Information dar, aber eben auch keine, die gegenüber der Karte mit der Staatsgrenze zusätzliches Wissen aktiviert. Zu erwarten war, dass etwa stadtsprachliche Konzepte deutlich werden, die auf einer geographischen Anbindung von Städten an Flüsse aufbauen, wie etwa im Falle Hamburgs und der Elbe. Dies ist allerdings nicht der Fall. Erstens werden solche Konzepte abgesehen von wenigen Ausnahmen nicht aktiviert, zweitens ist, wenn Stadtsprachen genannt werden, kein eindeutiger Bezug zum Flussnetz erkennbar. So wird z. B. Hamburgisch zwar einmal eingezeichnet, jedoch lässt sich weder die Nennung des Raums (Hamburgisch erscheint auch auf anderen Grundkarten) noch seine Verortung (am unteren "Ausgang" Schleswig-Holsteins) direkt aus dem auf der Grundkarte Flüsse gesetzten Stimulus ableiten. Lediglich in einem weiteren der nicht signifikanten Fälle lässt sich eine direkte Beeinflussung der Einzeichnungen durch die Flussvorgabe erkennen. Es handelt sich um den Sprachraum Fränkisch, der auf der FlüsseKarte zwischen Main und Donau angesiedelt wird, wohingegen die Verortungen auf den anderen Grundkarten deutlich diffuser ausfallen, selbst bei den Karten mit höherem Detailliertheitsgrad (Städte, Großstädte, Bundesländer, Kombination).

\footnotetext{
${ }^{33}$ Cf. Kap. 3.1.3.
} 


\subsubsection{Grundkarte Bundesländer}

Bundesländer $(\mathrm{N}=23)$ :

Bayerisch (20), Hessisch (20) > Berlinerisch (19), Schwäbisch (19) > Sächsisch (15) > Norddeutsch (11) > Kölsch (9)

Im Gegensatz zu den bisher besprochenen Kartentypen lässt sich auf der Grundkarte Bundesländer eine eindeutige Reaktion der Informanten auf den vorgegebenen Stimulus nachweisen. So ergibt sich durch die Einbeziehung der Bundesländergrenzen eine Binnenstrukturierung des Kartierungsgebietes, an dem die Schüler in deutlicher Weise ihre Eintragungen ausrichten. Da die Ländergrenzen vielfach nachgezeichnet werden, ist weithin nicht einmal von einer Orientierung, sondern von einer Übernahme der Ländergrenzen zu sprechen. Am deutlichsten zeigt sich dies an den Sprachräumen Hessisch, Schwäbisch, Berlinerisch und Bayerisch (cf. für Bayerisch die Abb. 5).

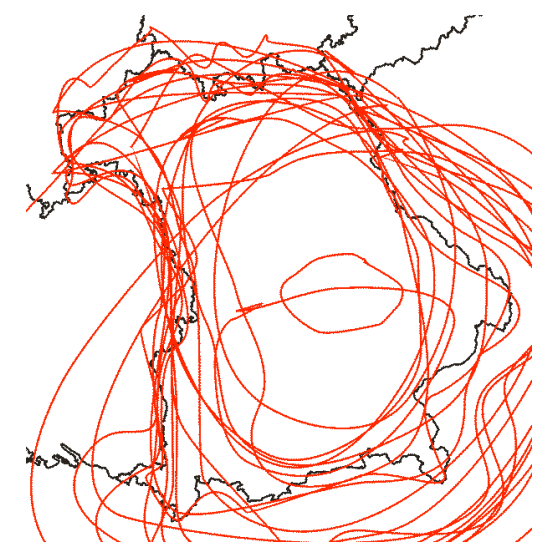

Abb. 5: Ausschnitt der Eintragungen für Bayerisch auf der Grundkarte Bundesländer

Hier sind die Verläufe der Sprachraumgrenzen mit denen der politischen Grenzen praktisch identisch. Eine Ausnahme unter den prominenten Sprachräumen bildet die Eintragung Norddeutsch, die, wie bereits gezeigt, topographisch motiviert ist und somit auch auf der Bundeslandkarte bestens integrierbar ist. Eine grundsätzliche Übernahme von Bundesländern (etwa Schleswig Holstein und Mecklenburg-Vorpommern) ist diesbezüglich nicht erkennbar (cf. Abb. 6). ${ }^{34}$

\footnotetext{
${ }^{34}$ Cf. Kap. 3.2.2.1. Gleichzeitig lassen sich aus den Daten Hinweise darauf ableiten, dass die konstante Verortung von Norddeutsch als Küstensprache neben der topographischen Orientierung auch konzeptuell motiviert ist. Cf. hierzu Kap 5.
} 


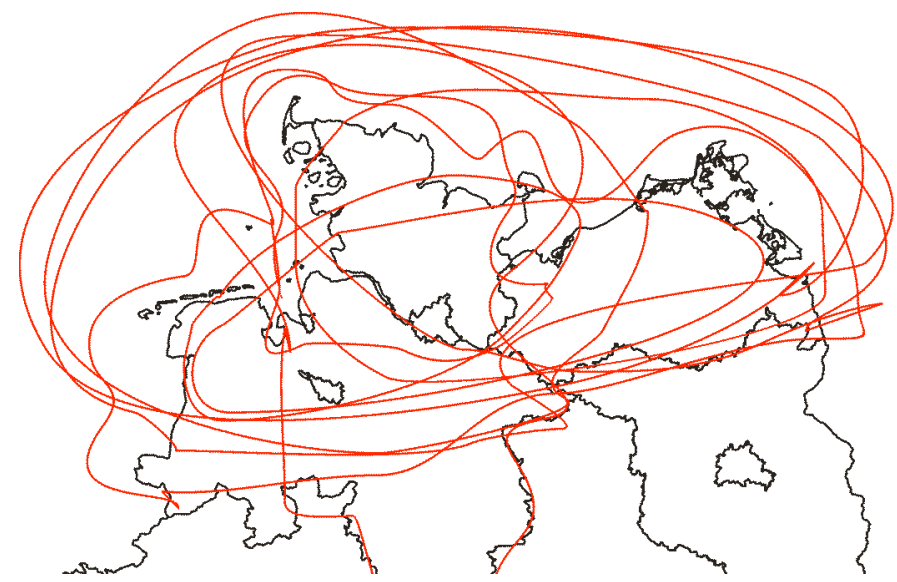

Abb. 6: Ausschnitt der Eintragungen für Norddeutsch auf der Grundkarte Bundesländer

Dieser norddeutsche Sonderfall deutet darauf hin, dass trotz aller Beeinflussung nicht unreflektiert sämtliche Bundesländer mit Sprachräumen gleichgesetzt werden. So weist der Kartentyp weitgehend dieselben signifikanten Sprachräume aus wie das Gesamtcluster. Der gesetzte Stimulus wirkt sich vor diesem Hintergrund nicht (!) auf die Nennung selbst, sondern eher auf die Verortung der genannten Sprachräume aus. Dieser Einfluss ist so stark, dass sich die für das Gesamtsample charakteristischen Flächentypen nur in Einzelfällen nachweisen lassen. Lediglich die Eintragungen für Bayerisch, Sächsisch und Kölsch entsprechen den vorgestellten Typen. Gerade die Konzeptualisierung des Sächsischen bedarf weiterer Ausführungen. Mit Blick auf die aus der Gesamtanalyse hervorgegangenen Sprachräume präsentiert sich der sächsische Sprachraum als ein sehr großflächiger, der nahezu das gesamte Gebiet der ehemaligen DDR überdeckt (cf. auch Auer 2004, 165). Ähnliches zeigt sich auch bei den anderen Kartentypen. Unter Berücksichtigung des Umstandes, dass der hier verwendete Stimulus eine Konzentration auf den politischen Raum Sachsens ermöglicht, kann die Konzeptualisierung des Sächsischen etwas genauer gefasst werden. Wie sich zeigt, ist der Kernraum für das Sächsische ebenfalls stark an den Bundeslandgrenzen Sachsens orientiert. Dennoch ist die Tendenz zur Gleichsetzung mit dem Gebiet der ehemaligen DDR klar erkennbar. Sächsisch ist also in der Wahrnehmung zumindest eines Teils der Informanten "das, was in der ehemaligen DDR gesprochen wurde". ${ }^{35}$ Dafür spricht auch, dass sich auf allen Grundkarten neben dem Sächsischen für den Osten Deutschlands - von Berlin abgesehen - lediglich ein weiterer signifikanter Sprachraum finden lässt, nämlich Ostdeutsch (cf. Kap. 3.2.2.5). Damit offenbart sich auf den ersten Blick eine fehlende Präsenz der ostdeutschen Sprachräume, die sich in der hier bereits angedeuteten Alternativbenennung von Sächsisch und Ostdeutsch niederschlägt.

Unter Berücksichtigung aller Ergebnisse ist festzustellen, dass die Angabe der Bundesländer zu Eintragungen führt, die wenige Rückschlüsse hinsichtlich mentaler Repräsentationen zulassen. Die Referenz auf ein Bundesland kann allenfalls als ein Hinweis darauf verstanden werden, dass in eben diesem Bundesland ein wie auch immer bezeichneter Dialekt vorkommt. In solchen Fällen dienen die politischen Grenzen zur Kennzeichnung regionalsprachlicher Prototypen.

\footnotetext{
35 Diese Definition lässt sich dabei auch umgekehrt lesen: "Auf dem Gebiet der ehemaligen DDR wurde/wird Sächsisch gesprochen." Diese Einschätzung der Schüler entspricht der Beobachtung, dass, abgesehen vom Berlinischen, Sächsisch die einzige regionalsprachliche Variante auf dem Gebiet der ehemaligen DDR ist, die medial bundesweit verbreitet wird.
} 


\subsubsection{Grundkarte Großstädte}

\section{Großstädte $(\mathrm{N}=23)$ :}

Bayerisch (23) > Hessisch (19) > Sächsisch (18), Schwäbisch (18) > Berlinerisch (16) > Norddeutsch (14) $>$ Kölsch (13) > Hochdeutsch (11)

Durch die Angabe der 15 deutschen Großstädte mit Einwohnerzahlen von über 800.000 wird - ähnlich wie durch die Grenzen der Bundesländer - eine Strukturierung des Kartenbildes vorgenommen. Zusätzlich werden den Informanten mit den Städtenamen Benennungen angeboten, die eine direkte Aktivierung von weniger spontanem Wissen erwarten lassen. Es wurde angenommen, dass sich dies in den Eintragungen in der Erhöhung des Anteils von stadtsprachlichen Konzepten niederschlägt. Jedoch wird diese Erwartung hinsichtlich der Anzahl der Nennungen lediglich teilweise erfüllt. Sieht man von den auch für andere Kartentypen signifikanten Stadtsprachen Berlinerisch und Kölsch ab, so finden sich zwar bei diesem Kartentyp auch vereinzelte Eintragungen, die sich auf bestimmte Großstädte beziehen, beispielweise orientiert an den Vorgaben Hamburg oder Frankfurt. Das Drittelkriterium weist jedoch keinen dieser Stadträume als signifikant aus. Wenn also solche Eintragungen vorgenommen werden, so haben sie individuellen Status. Dennoch ist der Einfluss des Stimulus evident. Er geht sogar so weit, dass auf Städte referiert wird, die gar nicht vorgegeben sind, wie etwa im Falle des Erhebungsortes Marburg. Es ist auffällig, dass diese Nennung, obwohl sie naheliegend scheint, auf keinem anderen Grundkartentyp vorkommt. Dem gegenüber steht allerdings, dass mit der Nennung Mainz der Fall einer anderen nicht vorgegebenen Stadt vorliegt, die jedoch auch auf anderen Grundkartentypen vorkommt. ${ }^{36}$ Was die als signifikant zu bewertenden Sprachräume anbelangt, so entsprechen sie denen des Gesamtsamples exakt.

Hinsichtlich der Verortungsstrategien für die eingezeichneten Sprachräume zeigt die Großstädte-Karte jedoch einen klaren Effekt: Für alle signifikanten Sprachräume fungiert eine der vorgegebenen Städte als eindeutiger Bezugspunkt und gleichzeitiger Lokalisationskern des eingezeichneten Sprachraums. "Kern" bedeutet im vorliegenden Fall, dass nahezu sämtliche für einen Sprachraum vorgenommenen Eintragungen die jeweilige Großstadt umschließen, ohne dass diese geometrisches Zentrum des Gesamtsprachraums sein muss, auch wenn sie es dessen ungeachtet auffallend häufig ist. Eine Bestätigung für das von Auer (2004) diskutierte Zentrum-Peripherie-Modell lässt sich insofern ableiten, als Sprachräume offensichtlich über Großstädte als konzeptuelle (nicht geographische!) Zentren identifiziert werden. Es liegt nahe anzunehmen, dass die Großstädte allein deshalb für die signifikanten Sprachräume zentral gesetzt werden, weil sie auf der Karte vorgegeben sind, also - abgesehen von der Staatsgrenze - den einzigen strukturierenden Stimulus darstellen, vergleichbar der Orientierung an den Bundesländergrenzen auf der Karte Bundesländer (Kap. 3.2.2.3). ${ }^{37}$ Die Ausdehnung der Umrandung des jeweiligen Kernraums ist relativ vage. Aus der Lage des Lokalisierungskerns lassen sich Rückschlüsse auf die konzeptuelle Strukturierung der einzelnen Sprachräume ziehen. So schließt etwa das Zentrum für Bayerisch München ein. Für das Hessische weisen die Eintragungen, ausgehend von dem Lokalisationskern um Frankfurt, eine deutliche Orientierung nach Norden und Osten auf. Den Informanten ist also die "Randlage" des subjektiven Sprachraumkerns Frankfurt innerhalb des Hessischen (bzw. innerhalb Hessens) durchaus bewusst. Ähnlich verhält es sich im Fall Hochdeutsch, bei dem Hannover - einem seit langem tradierten Volksglauben folgend - als Zentrum definiert wird. Damit wird zugleich ein Hinweis auf die häufig medial vermittelte und stereotype Repräsentation

\footnotetext{
${ }^{36}$ Mit jeweils einer Nennung kommen noch die Großstädte Düsseldorf und Stuttgart hinzu.

${ }^{37}$ Weitere Hinweise für diese Annahme, die zudem das Zentrum-Peripherie-Modell als zentrales Strukturierungsmerkmal für die Sprachräume relativieren, finden sich in den Ergebnissen für die Städte-Karte (cf. Kap. 3.2.2.5).
} 
sprachlicher Konzepte geliefert. Auch die Eintragungen für das Sächsische funktionieren in dieser Weise. Allerdings besteht das Zentrum hier mit Leipzig und Dresden gleich aus zwei Städten (cf. Abb. 7).

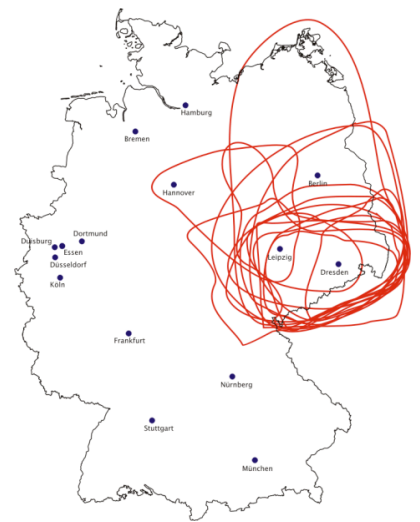

Sächsisch

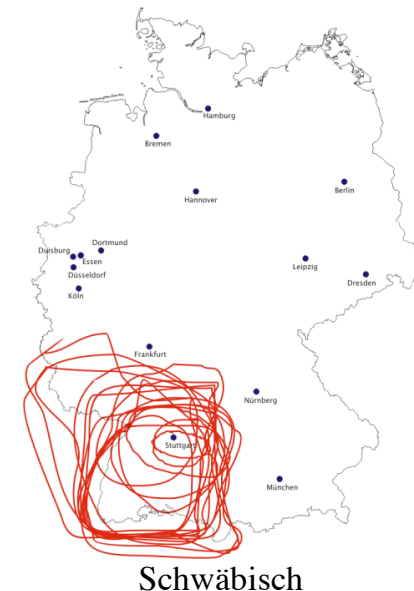

Schwäbisch

Abb. 7: Eintragungen für Sächsisch und Schwäbisch auf der Grundkarte Großstädte

Nicht zu verkennen ist in vielen Fällen auch eine Sekundärorientierung an der Staatsgrenze, wie z. B. die Eintragungen für Schwäbisch in Abb. 7 belegen, für das, ausgehend von Stuttgart als Lokalisationskern, die Sprachraumgrenze zumeist bis an die südwestliche Grenze der Bundesrepublik führt. Auch die Charakterisierung des um Hamburg angesiedelten Norddeutschen behält seine Charakteristik als "Sprache des Nordens".

Alles in allem führt die Angabe der Großstädte zu einem differenzierten Kartenbild. Ein Zirkeleffekt, wie er zunächst vermutet werden konnte, ist nicht nachweisbar. Da die signifikanten Nennungen mit denen der vorherigen Kartentypen weitgehend deckungsgleich sind, kann davon ausgegangen werden, dass die Informationsvorgabe sich nicht verzerrend auswirkt. Vielmehr wird eine konzeptuelle Konkretisierung erreicht, die den individuellen Repräsentationen näher zu kommen scheint, als es in den bisherigen Grundkartentypen der Fall war. Die Großstädte fungieren dabei als Prototypen (wie Stereotype) für den jeweiligen Sprachraum und aktivieren einen weiteren Aspekt des subjektiven Wissens der Informanten über den betreffenden Sprachraum.

\subsubsection{Grundkarte Städte}

Städte $(\mathrm{N}=25)$ :

Bayerisch (24) > Berlinerisch (20) > Hessisch (18) > Sächsisch (17) > Schwäbisch (16) > Kölsch (15) $>$ Hochdeutsch (12) > Norddeutsch (11) > Ostdeutsch (9)

Für den Grundkartentyp Städte war, da einzelne Städte gezielt in ein Gebiet integriert oder aus diesem ausgeschlossen werden können, von einer deutlichen Beeinflussung der Eintragungen durch den vorgegebenen Stimulus auszugehen. Vor allem war im Vergleich zu den bisherigen Grundkartentypen mit differenzierteren Grenzverläufen zu rechnen. In der Tat weist dieser Kartentyp mit insgesamt 102 Ortsnamen im Vergleich zur Großstädte-Karte eine deutlich höhere Informationsdichte auf. 38

Der erwartete Differenzierungseffekt lässt sich in den Karten deutlich nachweisen: Wurde der Lokalisationskern eines Sprachraums auf der Großstädte-Karte lediglich über eine einzelne Stadt repräsentiert (Ausnahme Sächsisch), so werden hier mehrere Städte in ein konzeptuelles Zentrum integriert. Als typisches Beispiel dienen die Eintragungen für Bayerisch, bei dem die

\footnotetext{
${ }^{38}$ Die Großstädte sind in dieser Gesamtanzahl enthalten.
} 
Großstädte-Karte eine klare Dominanz von München ausweist. Werden allerdings mehr Städte vorgegeben, so werden auch die Städte Augsburg, Landshut, Ingolstadt, Straubing, Rosenheim und Regensburg, gemeinsam mit München, als für das Sprachraumkonzept Bayerisch zentral angesehen und in den Lokalisationskern integriert. Bemerkenswerterweise liegt München in diesem komplexen Lokalisationskern nicht zentral, sondern am unteren Rand. Diese Beobachtung widerspricht dem am Beispiel der Karte Großstädte angesprochenen Zentrum-Peripherie-Modell (Kap. 3.2.2.4). Verstehbar ist diese Diskrepanz als Effekt der Informationsstruktur der Grundkarten und den damit verbundenen Konsequenzen für die Aktivierung von Sprachraumwissen. Es ist davon auszugehen, dass München, die größte und wichtigste Stadt in Bayern, als regionaler Prototyp fungiert und insofern konzeptuell für das Bayerische als zentral anzusetzen ist. Diese Funktion Münchens erweist sich anhand der Großstädte-Karte als evident. Durch die Vorgabe weiterer Städte jedoch scheint bei den Schülern ein weiterer Aspekt ihres individuellen sprachraumbezogenen Wissens aktiviert zu werden, der sowohl feiner differenziert als auch konzeptuell komplexer ist als es die Karte Großstädte ermöglicht. Da die Eintragungen in der Mehrzahl der Fälle nicht flüssig gezeichnet sind, sondern an den Einzelstädten absetzen, handelt es sich - im Gegensatz zur Karte mit den Großstädten - nicht um vage, sondern um reflektierte Grenzziehungen. Verfolgt man im Falle von Bayerisch, ausgehend vom Zentrum des Sprachraums, die Eintragungen der Informanten nach Norden und Westen weiter (cf. Abb. 8), so lassen sich mehrere, deutlich unterschiedene Ringe um das Zentrum beschreiben, die folgende Städte umfassen: 1. Ring: Ansbach, Nürnberg, Erlangen, Weiden, Amberg, Passau / 2. Ring: Bamberg, Bayreuth, Kempten / 3. Ring: Würzburg, Coburg, Schweinfurt, Hof, Memmingen / 4. Ring: Suhl, Ulm, Ravensburg. An den spezifischen Verläufen lässt sich aber nicht nur ablesen, dass die Informanten bestimmte Städte dem Bayerischen gezielt zuordnen, sondern auch, dass sie bestimmte Städte gezielt ausschließen, so etwa Reutlingen oder Stuttgart.

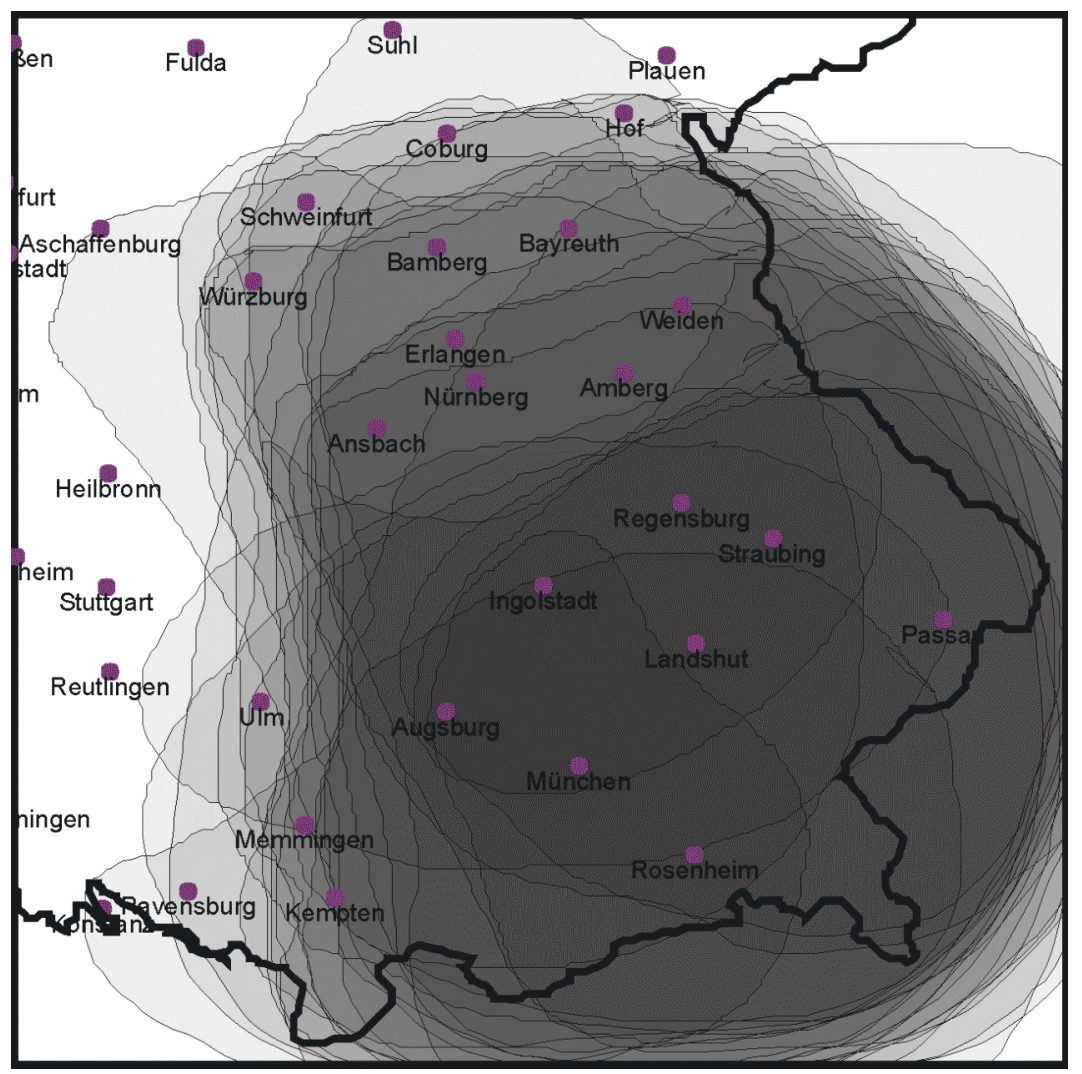

Abb. 8: Ausschnitt der Eintragungen für Bayerisch auf der Grundkarte Städte

Deutlich wird dieses Vorgehen auch am Beispiel der Nennung Sächsisch, wie Abb. 9, ein Detailausschnitt aus der Städte-Karte für das Sächsische, verdeutlicht. Ausgehend vom 
Lokalisationskern lässt sich zeigen, wie einzelne Informanten gezielt einzelne Städte in den Sprachraum mit einbeziehen, auch solche, die politisch bereits zu Thüringen (Gera, Jena, Erfurt, Eisenach) bzw. Sachsen-Anhalt (Halle, Dessau, Halberstadt, Madgeburg) gehören.

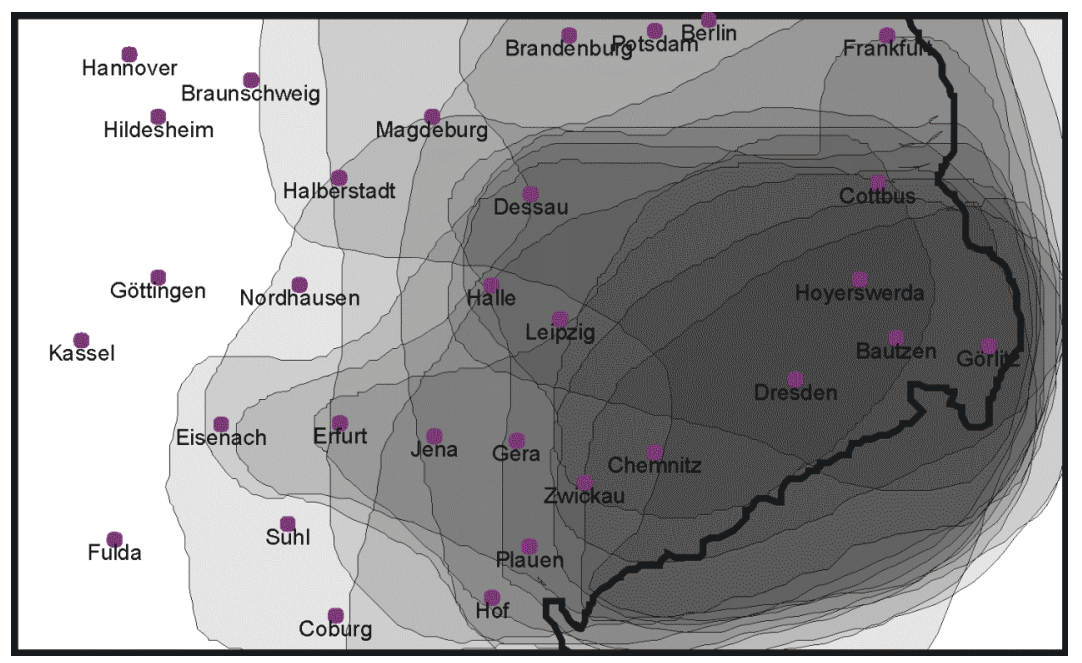

Abb. 9: Ausschnitt der Eintragungen für Sächsisch auf der Grundkarte Städte

Vergleichbare Strategien lassen sich auch für die übrigen prominenten Sprachräume dieses Kartentyps nachweisen. Weitere Hinweise auf den spezifischen Charakter der durch diesen Grundkartentyp stimulierten Angaben liefern auch die Eintragungen für die beiden stadtbezogenen Sprachräume Kölsch und Berlinerisch. Die Lokalisationskerne dieser beiden Räume umfassen jeweils lediglich die Stadt selbst. Darüber hinaus jedoch identifiziert ein Teil der Informanten zusätzlich Städte in der Umgebung mit dem jeweiligen Sprachraum. So werden auch die Städte Potsdam, Brandenburg, Stendal, Neuruppin, Eberswalde, Frankfurt/Oder sowie Neubrandenburg dem Berlinerischen zugeordnet. Für Kölsch sind dies wesentlich die Städte des Ruhrgebiets (auf der Karte vertreten durch Krefeld, Essen und Dortmund) sowie Düsseldorf, Münster, Siegen und Bonn. Über diese Verortungen werden hier solche Konzepte erfasst, in denen sprachlich und/oder außersprachlich "irgendwie" in Beziehung stehende Städte in der subjektiven Bewertung zu Sprachräumen verbunden werden. ${ }^{39}$ Insgesamt zeigt sich, dass mit der Städtekarte andere Konzepte erhoben werden als bei dem zuvor diskutierten Kartentyp mit den Großstädten. Der gesetzte Stimulus aktiviert ein äußerst differenziertes Sprachraumwissen. Im Gegensatz zur Karte mit den Großstädten werden hier keine lokalen Prototypen reflektiert, sondern Sprachlandschaften definiert.

Auch die zuvor bereits thematisierte Nennung Ostdeutsch, die hier abschließend diskutiert werden soll, fügt sich in dieses Bild (Kap. 3.2.2.3). Die für alle Grundkarten sichtbare Tendenz zur Gleichsetzung des Sächsischen mit dem Gebiet der ehemaligen DDR findet ihre Entsprechung in dem auf diesem Kartentyp signifikanten Sprachraum Ostdeutsch, der ebenfalls das gesamte Gebiet der ehemaligen DDR - ausgenommen Berlin - einschließt. Auffällig daran ist, dass beide Sprachräume, von einer Ausnahme abgesehen, alternativ eingezeichnet werden. Entweder findet sich in den Karten der Informanten ein Sprachraum Sächsisch oder ein Sprachraum Ostdeutsch, der jeweils wahlweise an Städten Sachsens oder eben einem größeren Gebiet innerhalb der ehemaligen DDR orientiert ist. Dieser Umstand deutet darauf hin, dass das Sächsische als sprachlich auffällige und medial verbreitete regionale Variante des Deutschen die Funktion eines - für diesen Kartentyp kleinräumig differenzierten - regionalen Prototyps wie Stereotyps übernimmt, in dem letztlich das

\footnotetext{
${ }^{39}$ Im Falle Berlins korrelieren die Eintragungen weniger mit politischen als vielmehr mit sprachlichen Beziehungen zwischen Berlin und Brandenburg. Dagegen korrelieren im Falle Kölns weniger die sprachlichen als vielmehr die kulturellen Beziehungen der Städte des Rhein-Ruhr-Raumes.
} 
mangelnde Wissen der Informanten über die Struktur des ostdeutschen Sprachraums zum Ausdruck kommt. ${ }^{40}$

\subsubsection{Grundkarte Kombination}

\section{Kombination ( $\mathrm{N}=26)$ :}

Bayerisch (26) > Hessisch (20) > Sächsisch (19) > Berlinerisch (17), Schwäbisch (17), Kölsch (17) $>$ Norddeutsch (14) > Hochdeutsch (11)

Die statistische Auswertung der Anzahl und Art von Sprachraumnennungen auf den einzelnen Grundkartentypen hat gezeigt, dass die Kombinationskarte im Vergleich die meisten Sprachraumnennungen bei größtmöglicher Variation evoziert, worin sie der Grundkarte Staatsgrenze gleicht. Diese statistische Ähnlichkeit wurde angesichts maximal unterschiedlicher Informationsdichte zwischen den beiden Kartentypen darauf zurückgeführt, dass sich auf der Karte Kombination die Orientierungsstrategien durch die Fülle an Einzelinformationen gegenseitig aufheben bzw. "behindern" und sich die Informanten nur auf bestimmte Arten von zusätzlichen Angaben auf der Grundkarte konzentrieren.

Bei eingehender Betrachtung der Eintragungen für diesen Grundkartentyp lässt sich nun feststellen, dass die Informanten die unterschiedlichen Verortungs- und Aktivierungsstrategien, die durch die verschiedenen Stimuli ausgelöst werden, individuell - und nach Sprachraum verschieden - in unterschiedlicher Weise nutzen. Dabei zeigt sich ein starker Einfluss der Bundesländergrenzen auf die Verortung der Sprachräume. Ein Großteil der Eintragungen orientiert sich an den politischen Grenzen, so etwa im Falle von Bayerisch, Berlinerisch, Hessisch, Sächsisch und Schwäbisch.

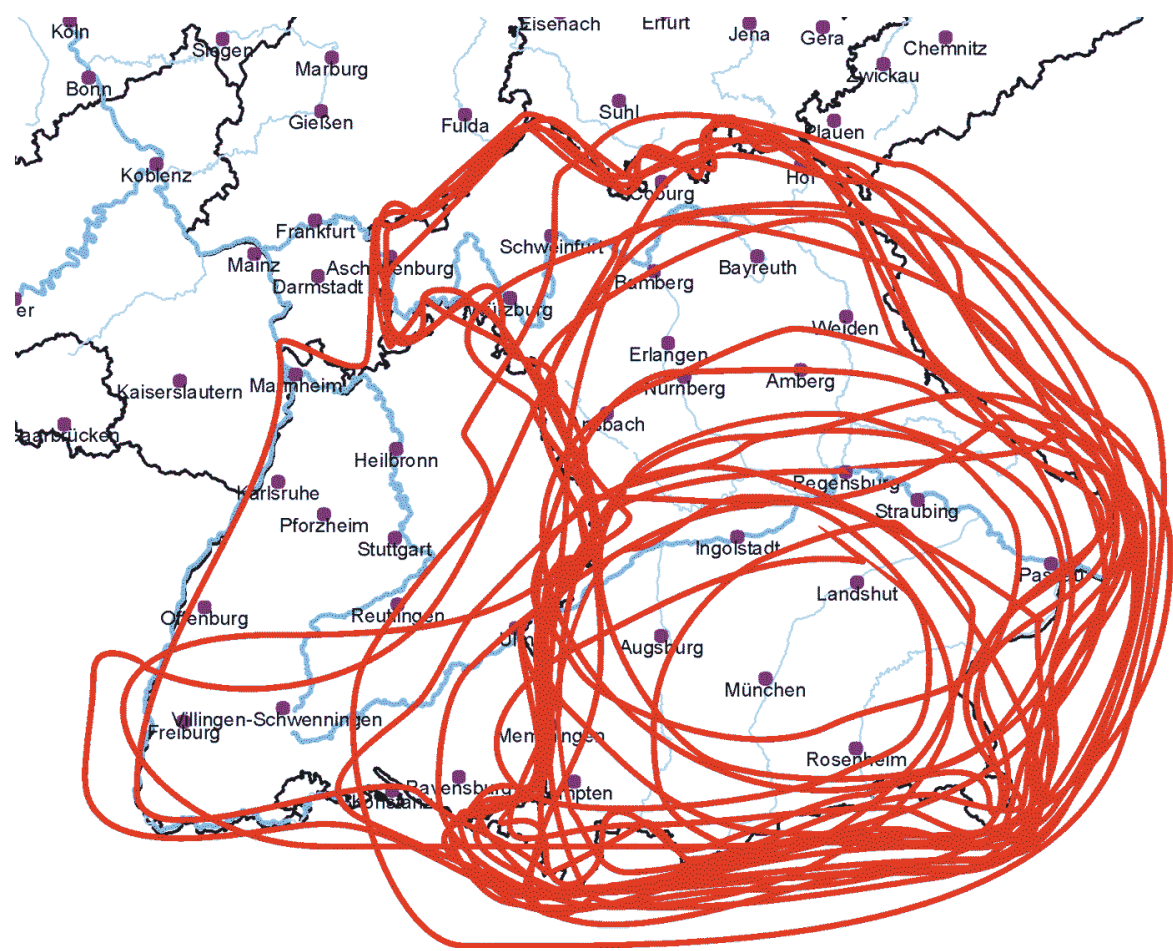

Abb. 10: Ausschnitt der Eintragungen für Bayerisch auf der Grundkarte Kombination

Innerhalb dieser Grenzen jedoch nehmen die Informanten im Einzelfall ähnlich differenzierende Abstufungen vor, wie sie die Städte-Karte hervorgebracht hat. Andere Stimuli haben

\footnotetext{
${ }^{40}$ Es steht $\mathrm{zu}$ erwarten, dass diese pauschale konzeptuelle Gleichsetzung bei einer Befragung an einer ostdeutschen Schule nicht aufträte. Cf. auch die Feindifferenzierungen des Sächsischen Sprachraums durch sächsische Informanten bei Anders (2007).
} 
dem gegenüber nur nachrangige Bedeutung. Insofern kann für den Kartentyp Kombination eine kognitive Hierarchie der Stimuli in Bezug auf die Lenkung der Verortungen angenommen werden. An erster Stelle stehen dabei eindeutig die Bundesländergrenzen. Werden sie auf der Karte vorgegeben, definieren sie die Bezugsdimension für die regionale Verortung. Darüber hinaus kann die Angabe von Städten weiterhin differenzierend wirken, im Vergleich zur Städte-Karte fallen diese Differenzierungen aber deutlich weniger markant aus. Dass diese Stimuli allerdings nicht den einzigen Anhaltspunkt für die Verortung der Sprachräume bilden, belegen die Eintragungen für die übrigen drei prominenten Sprachräume. So ist das Konzept "Sprache der Küste" für das Norddeutsche stabil, ungeachtet der Bundesländergrenzen und Städte auf der Grundkarte. Hier haben sich die Informanten allem Anschein nach am Küstenverlauf orientiert. Der stadtbezogene Sprachraum Kölsch lässt sich nach den vorliegenden Ergebnissen ebenso wenig wie Hochdeutsch auf einen speziellen Stimulus zurückführen.

Insgesamt gesehen liegt mit der Grundkarte Kombination ein Mischtyp vor, der individuell wie raumbezogen unterschiedliche Verortungsstrategien ebenso wie verschiedene konzeptuelle Aspekte des individuellen Sprachraumwissens aktiviert. Individuell ist dabei von einem Selektionsprozess in Bezug auf die dargebotenen Informationstypen auszugehen, der der Menge an Stimuli in der Karte zuzuschreiben ist. Leitend für die Eintragungen eines Informanten sind nicht alle, sondern jeweils ein dominanter Stimulus, der den Raum primär strukturiert. In Bezug auf die Sprachräume wiederum zeigt sich eine vergleichbare Hierarchie der Verortungsstrategien, in denen sich die erhobenen Teilaspekte der individuellen Sprachraumkonzeptualisierungen spiegeln.

\subsection{Repräsentanten der Sprachräume}

Im Anschluss an die Auswertung der Verortungen soll ergänzend nach in der Wahrnehmung der Schüler typischen Repräsentanten für die von ihnen genannten Sprachräume gefragt werden. ${ }^{41}$ Sieht man von individuellen Erfahrungen (Urlaub, Freunde etc.) ab, so ergeben sich klare Hinweise darauf, dass die eingetragenen Sprachräume vorwiegend mit stereotypen Sprachvorbildern in Zusammenhang stehen, also medial vermittelten regionalen Prototypen. Von den Schülern werden zumeist solche Politiker, Sportler oder in den Medien - d. h. vorwiegend dem Fernsehen - präsente Künstler (Komiker, Schauspieler) als typische Sprachraumvertreter benannt, die sprachlich stets durch eine regionale, aber an der Standardsprache orientierte Sprechweise charakterisiert werden können. Typische Nennungen stellen etwa Martin Schneider für Hessisch oder Edmund Stoiber für Bayerisch dar. ${ }^{42}$ Bei all diesen Nennungen fällt auf, dass in keinem Fall eine Person - aus linguistischer Sicht tatsächlich einen regionalen oder lokalen Dialekt repräsentiert. Es handelt sich in fast allen Fällen um regionale Akzente, die mehr oder weniger medial inszeniert werden. Daneben werden auch solche Prominente als typisch benannt, bei denen primär die Herkunft oder ein regionales Image und nicht etwa die sprachliche Auffälligkeit im Vordergrund zu stehen scheinen. ${ }^{43}$ Ein typischer Fall ist z. B. die Nennung von Otto Waalkes als Vertreter für Friesisch/Ostfriesisch/Norddeutsch. Weiterhin auffällig ist, dass von den sechs am häufigsten

\footnotetext{
${ }^{41}$ Die Frage auf der Rückseite des Erhebungsbogens lautete: "Sind Ihnen aus den eingezeichneten Sprachräumen prominente Personen bekannt und wenn ja, welche?"

42 Auch wenn die Prominentenfrage natürlich zu einigen Fehlnennungen führt, etwa Angela Merkel für Sächsisch, können nicht alle unzutreffenden Zuordnungen als Fehl- oder Scherznennungen klassifiziert werden. So liefern etwa die Nennungen Goethe und die Gebrüder Grimm als Repräsentanten für Hessisch Anhaltspunkte dafür, dass die Schüler den Begriff "Sprachraum" relativ offen und eher im Sinne eines Kulturraums definieren.

${ }^{43}$ Auch Preston (i. E.) erwähnt die gelegentlichen Angaben sogenannter kultureller Stereotype für Sprachräume in Mental Maps der USA.
} 
benannten Prominenten drei den Sprachraum Bayerisch repräsentieren ${ }^{44}$ : Edmund Stoiber/Bayerisch (61 Nennungen), Martin Schneider/Hessisch (48 Nennungen), Franz Beckenbauer/Bayerisch (27), Otfried Fischer/Bayerisch (22), Otto Waalkes/Norddeutsch/ Friesisch/Ostfriesisch (21), Gabi Köster/Kölsch (10). Dies wiederum ist als Hinweis darauf zu verstehen, dass Bayerisch im Vergleich zu allen übrigen Nennungen, den prominentesten und medial präsentesten - Fall eines Regionalsprachraums darstellt. ${ }^{45}$ Dagegen können die Schüler, abgesehen von wenigen Beispielen, kaum Angaben für den statistisch ebenfalls sehr prominenten Sprachraum Sächsisch machen. Genannt werden hier etwa Michael Ballack, Angela Merkel, Erich Honecker oder Sachsen-Paule ${ }^{46}$. Diese Angaben, die gleichzeitig Rückschlüsse auf die Interessen und Mediengewohnheiten der Schüler zulassen, zeugen wiederum von wenig differenziertem Wissen über das Sächsische. ${ }^{47}$

Die Eintragungen deuten somit darauf hin, dass die Informanten sich bei ihrer Strukturierung des Sprachraums zumeist auf standardnahe, häufig stereotype und im alltäglichen kommunikativen Umgang erfahrbare Konzepte regionaler Sprache stützen. Dialektnahe Varietäten scheinen dagegen nicht nur weitgehend außerhalb ihres Erfahrungsbereichs zu liegen. Sie leiten anscheinend auch nicht maßgeblich die jeweilige Verortung.

\section{$4 \quad$ Zusammenführung}

Das allgemeine Ergebnis der Untersuchung besteht im statistischen Beleg acht prominenter Sprachräume. Es handelt sich dabei um diejenigen Sprachraumkonzepte, die typischerweise im aktiven Wissen der Marburger Informanten verankert sind. In der Reihenfolge absteigender Prominenz sind dies nach Ausweis einer hierarchischen Clusteranalyse Bayerisch, Berlinerisch, Sächsisch, Schwäbisch, Hessisch, Hochdeutsch, Kölsch und Norddeutsch. Die raumstatistische Analyse der jeweiligen Lokalisationskerne führt zum Ergebnis einer besonders hohen Dichte der Verortungsflächen für Bayerisch, Hessisch und Sächsisch. Es lässt sich darüber hinaus eine Hierarchie der Dichterelationen ableiten, in der sich für die ersten vier Positionen (Bayerisch, Hessisch, Sächsisch, Schwäbisch) die Reihenfolge der häufigsten Nennungen wiederholt. Bei diesen Räumen korrelieren folglich Anzahl der Nennungen und Dichte des Lokalisationskerns. Hinsichtlich der Relationen dieser Lokalisationskerne zur Gesamtfläche der Eintragungen ergeben sich Indizien für die Abhängigkeit der Eintragungen von der geometrischen Gestalt des über die Grundkarte vorgegebenen Raumkörpers.

Bei einer weitergehenden Differenzierung nach unterschiedlichen Stimuli (= Grundkartentypen) bleiben die erwähnten acht Sprachräume der Clusteranalyse weitestgehend ähnlich verortet. Dennoch zeigen sich in der Analyse der Einzeleintragungen deutliche Effekte der speziellen Stimuli. Darauf aufbauend ergibt sich folgende Typisierung:

- Staatsgrenze: Der Stimulus bedingt eine topologische Anordnung der Sprachräume, die an der geometrischen Form der Staatsgrenze orientiert ist. Der Effekt ist in allen Grundkartentypen nachzuweisen. Die Positionen der Eintragungen sind interindividuell vergleichbar

\footnotetext{
${ }^{44}$ In die Auswertung gehen nur solche Nennungen ein, bei denen der Name des Prominenten eindeutig auf einen Sprachraum bezogen wird. Nennungen ohne Bezug zu einem Raum werden nicht berücksichtigt. Mehrfachnennungen waren möglich.

${ }^{45}$ Cf. hierzu auch die Ausführungen zum Sprachraum Hochdeutsch in Kap. 3.2.1.

${ }^{46}$ Ein durch seinen Auftritt in der Fernsehsendung "Big Brother" sowie einige Erotikproduktionen kurzzeitig bekannt gewordener Sachse.

${ }^{47}$ Implizit liefern diese Angaben damit auch ein Indiz für den schlechten Ruf des Sächsischen (cf. etwa Hundt 1992), indem sie zeigen, dass es kaum einen prominenten Repräsentanten für das Sächsische gibt, der seine Regionalität bewusst betont wie dies für Sprecher etwa aus Bayern normal ist.
} 
und interpretierbar. Aufgrund des heterogenen Antwortverhaltens ist auf eine besondere Spontaneität der Antworten zu schließen.

- Relief: Das geophysische Profil überfordert die Informanten, so dass das Sprachwissen nur ansatzweise aktiviert wird. Der Stimulus ist nicht nur zu unkonkret, sondern scheint Verortungen zu erschweren. Die Ergebnisse sind nur eingeschränkt vergleichbar und interpretierbar.

- Flüsse: Ein signifikanter Einfluss auf die Eintragungen ist nicht nachweisbar. Die Information ist in statistischem Sinne ohne Wirkung. Obwohl die Information zu unkonkret ist, erschwert sie die Verortungen nicht. Die Ergebnisse sind analog zum Stimulus Staatsgrenze zu sehen.

- Bundesländer: Es besteht eine extreme Beeinflussung durch den politischen Raum, der zu einer konzeptuellen Verengung führt. Die Eintragungen repräsentieren eine vage regionale Spezifizierung im Sinne des Ausweises regionaler Prototypen. Gleichwohl sind sie vergleichbar und interpretierbar.

- Großstädte: Der Stimulus beeinflusst in der Weise, dass die Großstädte als lokale Prototypen von Regionalsprachen konzeptualisiert werden. Die Aktivierung von Wissen unterschiedlichen Spontaneitätsgrades wird ermöglicht. Die Ergebnisse sind auffallend differenziert, sie sind vergleichbar und interpretierbar.

- Städte: Dieser Stimulus ermöglicht in besonderem Maße den Ausweis konzeptuell fassbarer Sprachlandschaften. Es werden keine Prototypen, sondern individuell definierte Sprachlandschaften ausgewiesen. Die Aktivierung von Wissen unterschiedlichen Spontaneitätsgrades wird ermöglicht. Die Ergebnisse sind vergleichbar und interpretierbar.

- Kombination: Die Heterogenität des Antwortverhaltens deutet auf eine hohe Spontaneität des aktivierten Wissensbestands hin. Die Kombinationskarte führt zur selektiven Auswahl und gegenseitigen Aufhebung einzelner Stimuli. Vor allem die Bundesländergrenzen wirken dominant. Der differenzierende Effekt des Städtestimulus ist vorhanden, aber stark eingeschränkt. Damit ist - etwa verglichen mit der Staatsgrenzenkarte - mit einem Verzerrungseffekt hinsichtlich der Verortungen zu rechnen. Wenngleich damit die interindividuelle Vergleichbarkeit eingeschränkt ist, sind die Ergebnisse dennoch interpretierbar.

Deutlich zu unterscheiden sind unterschiedliche Grade an Spontaneität, mit denen die Eintragungen vorgenommen werden. Den Eintragungen auf der Karte mit der Staatsgrenze ist diesbezüglich ein besonders hohes $\mathrm{Ma} 3$ auszuweisen. Insofern ist die Art der stimulierten Information abhängig von der Menge und Qualität der auf der Grundkarte dargebotenen Information. Es kann angenommen werden, dass die verschiedenen Grundkarten, die hier nach aufsteigender Informationsdichte und -konkretheit sortiert wurden, den Prozess der Aktivierung unterschiedlicher Aspekte eines komplexen Sprachraumkonzepts abbilden, von groben regionalen Verortungen über regionalsprachliche Prototypen und regionale Prototypen bis hin zu fein differenzierten Konzeptualisierungen. Ein zu hohes Maß unterschiedlicher Stimuli dagegen setzt spezielle Eigenschaften außer Kraft, wie die Karte Kombination eindeutig zeigt. In ähnlicher Weise funktioniert die Karte mit dem Relief, deren detaillierte physische Information die Informanten überfordert.

Grundsätzlich erweist sich damit die wissenschaftliche Eignung einer Grundkarte als vom Erkenntnisinteresse abhängig. Sollen spontane Wissensbestände hinsichtlich der bekannten regionalsprachlichen Räume erfragt werden, empfiehlt sich die Staatsgrenze-Karte, da sie abgesehen vom geometrischen und auch konzeptuellen Bezugsrahmen "Deutschland" keine weiteren Vorgaben bietet und also jeder genannte Sprachraum als weithin aktive Wissensleistung des Probanden verstanden werden kann. Es lässt sich ablesen, wo die 
Informanten einen Sprachraum innerhalb der Staatsgrenze platzieren und wie die Lage der Sprachräume zueinander eingeschätzt wird. Für eine Untersuchung möglichst exakter Grenzverläufe allerdings eignet sich dieser Kartentyp kaum, da er keinerlei Orientierung über die geometrische Form hinaus ermöglicht und daher die eingezeichneten Räume lediglich als ungefähre Regionszuschreibungen verstanden werden müssen. Besteht dagegen das Untersuchungsinteresse vorrangig in einer möglichst exakten Verortung der bekannten Sprachräume, scheint die Karte mit den detaillierten Städteangaben sinnvoll zu sein. Hinsichtlich der für den vorgeführten Effekt notwendigen Einheiten hat sich das verwendete Städteraster mit 102 Städten als angemessen erwiesen. Zu fragen wäre an dieser Stelle, wo die Grenze dieses Aktivierungsprozesses anzusetzen ist, ab welchem Detailliertheitsgrad der Karteninformation also die Informanten nicht mehr weiter differenzieren können bzw. überdifferenzieren. $\mathrm{Zu}$ erwarten wäre, und die Ergebnisse der Kleinraumstudie von Anders (2008) zum Sächsischen deuten - ungeachtet ihrer eingeschränkten Vergleichbarkeit - darauf hin, dass die Informanten ab einem bestimmten Detailliertheitsgrad nicht mehr regionale Gemeinsamkeiten sondern kleinräumige (und häufig objektiv nicht vorhandene) Differenzen kartieren. Wo aber dieser Umschlagpunkt anzusetzen ist, muss offen bleiben. In diesem Zusammenhang ist ebenfalls die grundsätzliche Fähigkeit von Sprechern zu berücksichtigen, kleinräumige Differenzierungen anhand von akustischen Stimuli vorzunehmen (cf. Purschke 2008).

Aufbauend auf diesem grundlegenden Ergebnis lassen sich aus den Daten Hinweise auf allgemeine und spezielle Konzeptualisierungen von Sprachräumen ableiten. Es darf angenommen werden, dass sich das vorgefundene Sprachwissen auf der Objektebene vorwiegend aus einem salienten Merkmalsbestand einer regional gefärbten Standardsprache konstituiert, der medial transportiert wird (Fernsehen, Internet). Insofern ist eine enge Anbindung des Wissensbestandes an den kommunikativen Alltag der Informanten vorauszusetzen. Es kann nicht überraschen, dass die Verortungen dieser Räume in einem allgemeinen Sinne Ähnlichkeiten mit der linguistischen Strukturierung der deutschen Regionalsprachen aufweisen. Mitunter scheinen die Räume in Verbindung mit städtischen Zentren zu stehen. Dies dürfte daher rühren, dass sich die Städte sowohl räumlich als auch sprachlich ideal als Identifikations- und Orientierungspunkte eignen. Ebenso sind aber auch Referenzen auf politische, soziale, kulturelle oder topographische Räume erkennbar. Damit ist die außersprachliche Orientierung angesprochen.

Typischerweise referieren die Nennungen auf unterschiedliche Einflussfaktoren. Dass nicht selten politischer Raum und Sprachraum gleichgesetzt werden (wie im Falle des Bayerischen), bedeutet nicht, dass Sprache unmittelbar an politische Bedingungen gekoppelt wäre. Vielmehr ist dies ein Effekt der Gleichsetzung von politischer Herkunftsregion eines individuell wirkenden Repräsentanten mit seiner geographischen Herkunft, die über den politischen Raum definiert wird. Das Konzept Sprachraum wird dann - teils aus Mangel an alternativem Wissen, teils beeinflusst durch die suggestive Kraft der Vorlage - mit eben diesem politischen Raum gleichgesetzt. ${ }^{48}$

Insgesamt lassen sich die eingetragenen Sprachräume vier unterschiedlichen Konzepttypen zuordnen:

\footnotetext{
${ }^{48}$ In vorliegenden Studien zum Japanischen finden sich starke Übereinstimmungen von Sprachräumen und Schulbezirken (cf. Mase 1964a und 1964b, Nomoto 1963), aber auch politisch-administrativen Räumen (cf. Sibata 1959 sowie zusammenfassend Preston (i.E.)). Auch in den Studien zu den USA werden sprachliche Raumstrukturen immer an den (vorgegebenen) Bundesstaaten orientiert. Inoue (1996) gibt für seine Untersuchung in Großbritannien an, dass die Informanten sich offenbar an geographischen Binnengliederungen orientieren, die sie von Karten aus der Schulzeit oder aus den Medien, z. B. Wetterkarten, kennen.
} 
- Konzepttyp 1 lässt sich über einen eindeutigen Bezug zu einem geographisch-politischen Raum definieren. Die Verortung der betreffenden Sprachräume erfolgt in der Regel kartenunabhängig und konstant. Beispiele hierfür sind etwa Bayerisch und das Hessisch.

- Konzepttyp 2 beschreibt Räume, die einen eindeutigen Bezug zu einer Stadt, einem erweiterten Stadtraum oder einem Städteverbund aufweisen, etwa Berlinerisch oder der Sprachraum Kölsch.

- Konzepttyp 3 stellt eine komplexe Mischform dar, bei der sich mehrere, von den Informanten nur teilweise differenzierte Räume überlagern. Diese Räume sind konzeptuell (Norddeutsch = Küstensprache) oder geographisch (Kölsch, Rheinisch, Ruhrpott, Pfälzisch, Rheinländisch $=$ Mitte Westen) an eine bestimmte, in ihrem Grenzverlauf jedoch nicht eindeutige Kartenregion gebunden. Der Unterschied zwischen Norddeutsch und dem "mittleren Westen" besteht dabei in dem Umstand, dass das Norddeutsche konstant regional verortet und bezeichnet wird, wohingegen das westlich der Kartenmitte liegende Areal durch verschiedene, sich räumlich wie konzeptuell überlagernde Eintragungen/Benennungen strukturiert ist. Unter diesen Typ ist auch der Fall Sächsisch/Ostdeutsch zu fassen.

- Konzepttyp 4 dient der Erfassung des Sonderfalls Hochdeutsch, das lediglich eine Negativverortung aufweist und dessen konzeptuelle Füllung insofern schwerer zu fassen ist, als sie in viel stärkerem Maße als bei den übrigen Sprachräumen von der individuellen Wahrnehmung und dem Bewusstsein für die eigene Regionalität abhängt.

Anhand dieser vier Konzepttypen lassen sich alle von den Informanten vorgenommenen Eintragungen beschreiben, auch jene, die in Bezug auf Nennung und Verortung unterhalb der statistischen Signifikanz liegen. ${ }^{49}$

\section{$5 \quad$ Ausblick}

Ausgehend von diesen Konzepttypen, deren Beleg zunächst ein sekundäres Ergebnis der methodischen Beschäftigung mit stimulusabhängigen Projektionsverfahren für mentale Sprachraumrepräsentationen darstellt, soll abschließend versucht werden, die mit den genannten und verorteten Sprachräumen verbundenen Konzepte wenigstens ansatzweise zu charakterisieren. Tiefergehende Analysen, die zugleich die konzeptuelle Motivation und Füllung der signifikanten Sprachraumnennungen hinterfragen, müssen weiteren Arbeiten vorbehalten bleiben. Dennoch lassen sich aus den vorliegenden Ergebnissen Hinweise ableiten, die illustrieren, wie die Repräsentation von sprachraumbezogenem Wissen strukturiert sein könnte. Am Beispiel der Sprachräume Sächsisch und Ostdeutsch (Kap. 3.2.2.3 und 3.2.2.5) sowie Hochdeutsch (Kap. 3.2.1) konnte dies bereits ansatzweise gezeigt werden. Eine ähnliche Überlagerung unterschiedlicher, zumeist stereotyper Aspekte von Sprachraumwissen lässt sich auch für andere Räume zeigen, am deutlichsten für die Nennungen, die den Konzepttypen 3 und 4 zugeordnet wurden. Für die übrigen, den Konzepttypen 1 und 2 zugrunde liegenden Sprachräume sind die Nennungen zumeist orientiert an einem medial verbreiteten regionalen Sprachprototyp, dessen Lokalisation weitgehend mit geographisch-politischen Bedingungen korreliert, ohne von diesen motiviert zu sein. Die Zuschreibung der Räume ist in diesen Fällen, von wenigen auf Teilräume bezogenen Untertypen (Südbairisch, Tiefsächsisch) abgesehen, konsistent und eindeutig.

Anders liegt der Fall etwa für den Sprachraum Norddeutsch, der von den Informanten gleichgesetzt wird mit "Sprache an der Küste". Damit besteht insofern ein konzeptueller Unterschied zu den anderen Angaben, als hier eine Sprachlandschaft mit einer konkreten topographischen Besonderheit des politischen Raumes in Verbindung gebracht und auf eine

${ }^{49}$ Ausgenommen sind dabei Scherzeintragungen wie "Assi-Slang", für deren Nennung nicht primär regionalsprachliche sondern soziale Faktoren entscheidend sind. 
einfache Formel heruntergebrochen wird: Norddeutsch $=$ das, was man an der Küste spricht. Das heißt umgekehrt: Die Küstenregion in Deutschland zeichnet sich durch eine eigene Sprache aus. Was die Benennung dieser Sprache angeht, bestehen Unterschiede zwischen den Informanten, die dabei gleichzeitig Hinweise auf die konzeptuelle Füllung eines umfassenderen Sprachraumkonzepts "Küstensprache" liefern. So findet sich in den vorliegenden Daten die Nennung Norddeutsch neben anderen wie Friesisch oder Plattdeutsch..$^{50}$ Diese wurden in der Analyse zunächst getrennt betrachtet, da nicht ausgeschlossen werden konnte, dass damit unterschiedliche Konzepte angesprochen sind. In der Tat jedoch sind die Eintragungen weitgehend deckungsgleich, so dass die Kartenbilder nahezu identisch sind. Die Angabe Friesisch (und auch Plattdeutsch) ist demnach als Teil des größeren Sprachraumkonzepts "Küstensprache" zu verstehen. Zwar finden sich Karten, auf denen diese Räume gleichzeitig ausgewiesen und regional differenziert werden, immer jedoch sind diese Räume entlang der Küstenlinie Norddeutschlands eingezeichnet. Zudem gibt es für diese Region häufige Doppelbenennungen von Räumen (z. B. Norddeutsch/Friesisch), die darauf hindeuten, dass diese Sprachräume Konstituenten desselben komplexen Konzeptes sind. Einen weiteren Hinweis auf die konzeptuelle Überlagerung dieser Sprachraumnennungen kann in dem Umstand gesehen werden, dass die Schüler den Komiker Otto Waalkes als - nahezu einzigen - prominenten Repräsentanten für alle drei Räume angeben. Insofern kann für den Sprachraum Norddeutsch eine konzeptuelle Motivation angenommen werden, die neben sprachlichen Prototypen topographische Aspekte sowie regional-mediale Stereotype vereint.

Als abschließendes Beispiel soll in diesem Zusammenhang die Kartenregion dienen, der als einziger kein eindeutiger (und nur ein kleinräumiger prominenter!) Sprachraum zugeordnet wird, die also auch in der Zusammenschau der Verortungen für alle signifikanten Sprachräume weitgehend unbelegt bleibt. Dieser westliche Teil der Kartenmitte, der politisch im wesentlichen die Bundesländer Nordrhein-Westfalen, Rheinland-Pfalz sowie das Saarland umfasst, wird von den Schülern nicht eindeutig benannt oder durch Eintragungen strukturiert. Stattdessen finden sich wechselnde Bezeichnungen, die sich auf mehrere verschiedene, sich konzeptuell wie geographisch teilweise überlagernde Räume beziehen: Kölsch (82), Ruhrpott (36), Pfälzisch (33), Saarländisch (30), Rheinisch/Rheinländisch (25).51 Auffällig daran ist, dass sich die jeweiligen Verortungen dieser genannten Sprachräume teilweise deutlich überschneiden. Dabei ist allerdings, anders als im Fall Norddeutsch, nicht davon auszugehen, dass die Informanten sich an einem komplexen, topographisch-sprachlich motivierten Konzept orientieren, sondern dass in diesen Benennungen das mangelnde Wissen der Informanten über die sprachliche Struktur dieser Region Deutschlands zum Ausdruck kommt. Zudem orientieren sich die Benennungen an den politischen Bezeichnungen der korrespondierenden Räume. Zwar ist den Schülern bewusst, dass diese Region durch unterschiedliche wie unterscheidbare regionale Varianten gekennzeichnet ist - häufig werden neben Kölsch weitere Sprachräume in einer Karte angegeben -, von denen manche darüber hinaus zumindest ansatzweise medial verbreitet werden, was sich etwa in der Nennung der Komikerin Gabi Köster als Repräsentantin für das Kölsche zeigt. ${ }^{52}$ Unsicher allerdings sind die Schüler hinsichtlich der Verortung dieser Nennungen. ${ }^{53}$ Insofern scheint es sich, anders als beim Norddeutschen, um ein komplexes Sprachraumkonzept zu handeln, das mehrere differenzierbare Sprachräume umfasst, die sich konzeptionell wie geographisch nur teilweise

50 Plattdeutsch ist für keinen Grundkartentyp ein prominenter Sprachraum, allerdings weisen die Eintragungen für diesen Sprachraum eine nahezu identische räumliche Charakteristik auf wie die Eintragungen für Norddeutsch oder Friesisch.

${ }^{51}$ Die Ziffern in der Klammer beziehen sich auf die Häufigkeiten der Nennung im Gesamtsample.

52 Von dieser Nennung abgesehen finden sich lediglich vereinzelte Zuschreibungen von Prominenten zu den genannten Räumen, etwa Herbert Grönemeyer für Ruhrpott oder Rainer Calmund für Kölsch.

${ }^{53}$ Es ist davon auszugehen, dass diese geographische Unsicherheit der Informanten bei einer Datenerhebung etwa in Köln für diesen Raum zumindest teilweise aufgehoben würde. 
überlagern, deren Verortung auf der Karte aber nicht eindeutig ist und sich an groben Charakteristika der Kartierungsgrundlage orientiert ("Ausbuchtung nach Westen in der Kartenmitte").

Es bleibt abzuwarten, ob und inwieweit diese Ergebnisse durch weitere Untersuchungen ihre Bestätigung finden oder weiter differenziert werden müssen. Auch die Frage, ob sich für weitere Sprachlandschaften des Deutschen ähnliche Beobachtungen hinsichtlich der Verortung und Konzeptualisierung von Sprachwissen machen lassen, bleibt vorerst unbeantwortet. Ungeachtet dieser noch ausstehenden methodischen wie inhaltlichen Vertiefung zeigt die vorliegende Studie, dass das Wissen über die Regionalsprachen des Deutschen und ihren regionalen wie überregionalen Stellenwert durch die Untersuchung kognitiver Repräsentationen in entscheidender Weise erweitert werden kann.

\section{Literatur}

Anders, Christina (2008): "Mental Maps linguistischer Laien zum Obersächsischen". In: Christen, Helen/Ziegler, Evelyn (ed.): Sprechen, Schreiben, Hören. Zur Produktion und Perzeption von Dialekt und Standardsprache zu Beginn des 21. Jahrhunderts. Wien: 203229.

Auer, Peter (2004): "Sprache, Grenze, Raum". Zeitschrift für Sprachwissenschaft 23: 149180.

Diercks, Willy (1988): "Mental Maps. Linguistisch-geografische Konzepte". Zeitschrift für Dialektologie und Linguistik 55: 280-305.

Downs, Roger/Stea, David (1973): Image and Environment. Cognitive Mapping and Spatial Behavior. Chicago.

Gessinger, Joachim (2000): "Überlegungen zu einer Sprachgeschichte des BrandenburgBerlinischen Raums". Niederdeutsches Jahrbuch 123: 57-83.

Gould, Peter/White, Rodney ( $\left.{ }^{2} 1986\right)$ : Mental maps. Boston/London/Sydney.

Hofer, Lorenz (2004): "Sprachliche und politische Grenzen im (ehemaligen) Dialektkontinuum des Alemannischen am Beispiel der trinationalen Region Basel (Schweiz) in Karten von SprecherInnen". Linguistik Online 20: 23-46.

Hundt, Markus (1992): Einstellungen gegenüber dialektal gefärbter Standardsprache. Eine empirische Untersuchung zum Bairischen, Hamburgischen, Pfälzischen und Schwäbischen. Stuttgart. (= Zeitschrift für Dialektologie und Linguistik, Beihefte 78)

Hundt, Markus/Anders, Christina (i.E.): "Regionale Differenzierung. Alltagsdialektologie und Dialektgeschichte. Sektionsbericht". In: Henn-Memmesheimer, Beate (ed.): Tagungsband des 41. Linguistischen Kolloquiums in Mannheim vom 06.-09. September 2006.

Inoue, Fumio (1996): "Subjective Dialect Division in Great Britain". American Speech 71: $142-162$.

Kitchin, Rob (1994): "Cognitive Maps.. What Are They and Why Study Them?" Journal of Environmental Psychology 14: 1-19.

Long, Daniel/Onishi, Isao (ed.) (o. J.): Online Perceptual Dialect Atlas. http://nihongo.human. metro-u.ac.jp/ long/maps/perceptmaps.htm, Stand 17.01.2008.

Lynch, Kevin (1960): The Image of the City. Cambridge.

Mase, Yoshio $\left(1999^{\mathrm{a}}\right)$ : "Dialect Consciousness and Dialect Divisions. Examples in the Nagano-Gifu Boundary Region". In: Preston, Dennis (ed.): Handbook of Perceptual Dialectology. Amsterdam/Philadelphia: 71-100. [Übersetzung von Mase, Yoshio (1964 $)$ : "Hôgen ishiki to hôgen kukaku". Nihon hôgen kenkykai: 270-302].

Mase, Yoshio (1999 $)$ : "On Dialect Consciousness. Dialect Characteristics Given by Speakers". In: Preston, Dennis (ed.): Handbook of Perceptual Dialectology. Amsterdam/Philadelphia: 101-114. [Übersetzung von Mase, Yoshio (1964 $4^{\mathrm{b}}$ : "Hôgen 
ishiki ni tsuite: Washa no genkyûshita hôgenteki tokuchô". Nagano-ken Tanki Daigaku Kiyô 18: 1-12].

Montgomery, Christopher (2007): Northern English dialects. A perceptual approach. Ph. D. dissertation, University of Sheffield.

Niedzielsky, Nancy/Preston, Dennis (2000): Folk Linguistics. Berlin/New York. (= Trends in Linguistics. Studies and Monographs 122)

Nomoto, Kikuo (1999): "Consciousness of Linguistic Boundaries and Actual Linguistic Boundaries". In: Preston, Dennis (ed.) (1999): Handbook of Perceptual Dialectology. Amsterdam/Philadelphia: 63-70. [Übersetzung von Nomoto, Kikuo (1963): "Kotoba ni ishiki no kyôkai to jissai no kyôkai". Jinruikagaku 15: 271-281].

Preston, Dennis R. (ed.) (1999): Handbook of Perceptual Dialectology. Amsterdam/ Philadelphia.

Preston, Dennis (i. E.): "Perceptual dialectology. Mapping the geolinguistic spaces of the brain". Erscheint in: Lameli, Alfred/Kehrein, Roland/Rabanus, Stefan (ed.): Language Mapping. An international handbook. Berlin, New York. (= Handbücher zur Sprach- und Kommunikationswissenschaft. Language and Space 2.)

Purschke, Christoph (2008): "Regionalsprachlichkeit im Hörerurteil". In: Christen, Helen/ Ziegler, Evelyn (ed.): Sprechen, Schreiben, Hören. Zur Produktion und Perzeption von Dialekt und Standardsprache zu Beginn des 21. Jahrhunderts. Wien: 183-203.

Purschke, Christoph (i. V.): Regionalsprachliche Grenzen im Hörerurteil [Arbeitstitel]. Marburg. [Dissertationsprojekt].

Schmidt, Jürgen Erich/Herrgen, Joachim (i. V.): Sprachdynamik. Eine Einführung in die moderne Regionalsprachenforschung. Marburg.

Schmitt, Ernst Herbert (1992): Interdialektale Verstehbarkeit. Eine Untersuchung im Rheinund Moselfränkischen. Stuttgart. (= Mainzer Studien zur Sprach- und Volksforschung 18).

Sibata, Takesi (1999): "Consciousness of Dialect Boundaries". In: Preston, Dennis (ed.) (1999): Handbook of Perceptual Dialectology. Amsterdam/Philadelphia: 39-62. [Übersetzung von Sibata, Takesi (1959): "Hôgen kyôkai no ishiki". In: Gengo Kenkyû 36, $1-30]$.

Siebenhaar, Beat (2000): Sprachvariation, Sprachwandel und Einstellung. Der Dialekt der Stadt Aarau in der Labilitätszone zwischen Zürcher und Berner Mundartraum. Stuttgart. (= Zeitschrift für Dialektologie und Linguistik, Beihefte 108).

Stickel, Gerhard/Volz, Norbert (1999): "Meinungen und Einstellungen zur deutschen Sprache. Ergebnisse einer bundesweiten Repräsentativerhebung". Mannheim. (= amades - Arbeitspapiere und Materialen zur deutschen Sprache 2/99)

Tolman, Edward (1948): "Cognitive Maps in Rats and Men". The Psychological Review 55: 189-208. 